\title{
Quantum States: An Analysis via the Orthogonality Relation *
}

\author{
Shengyang Zhong \\ Institute of Foreign Philosophy, \\ Department of Philosophy and Religious Studies, \\ Peking University, \\ Beijing, China \\ zhongshengyang@163.com
}

\begin{abstract}
From the Hilbert space formalism we note that five simple conditions are satisfied by the orthogonality relation between the (pure) states of a quantum system. We argue, by proving a mathematical theorem, that they capture the essentials of this relation. Based on this, we investigate the rationale behind these conditions in the form of six physical hypotheses. Along the way, we reveal an implicit theoretical assumption in theories of physics and prove a theorem which formalizes the idea that the Superposition Principle makes quantum physics different from classical physics. The work follows the paradigm of mathematical foundations of quantum theory, which I will argue by methodological reflection that it exemplifies a formal approach to analysing concepts in theories.
\end{abstract}

Keywords: Orthogonality relation, Mathematical foundations of quantum theory, Quantum logic, Concept analysis

\section{Background and Introduction}

\subsection{Mathematical Foundations of Quantum Theory}

Developed since the beginning of the 20th century, quantum theory is a new theoretical framework in physics which has proved to be able to describe microscopic physical phenomena with amazing accuracy. Along with its development in theory and success in application, quantum theory has been observed to have many novel characters which make it quite different from classical physics. In 1936, G. Birkhoff and J. von Neumann [10] pointed out a novel character of quantum theory from the perspective of logic. They showed that, while, as had been well known, the properties of a classical system form a Boolean algebra, those of a quantum system do not.

*Supported by NSSFC (No. 20CZX048). 
This observation leads to a lot of attention and interest in the lattice formed by the (testable) properties ${ }^{1}$ of a quantum system, although this happened many years after the publication of the paper. Researches about such lattices form a field called quantum logic. References include [12, 26, 29]. Quantum logic has now been widely acknowledged as an important kind of non-classical logic.

The paper [10] also inspires another line of research, which shares some technical results with quantum logic but has a slightly different objective. It is carried out by G. Mackey, J.M. Jauch, D.J. Foulis, C. Piron, C.H. Randall, D. Aerts, A. Wilce, etc. This field of research is usually called mathematical foundations of quantum theory $(M F Q T)^{2}$, but other names are also used by different authors according to their understanding of the subject. For example, it is called 'quantum axiomatics' in [2] and 'rational reconstruction of quantum mechanics' in [30]. Sometimes it is also called 'the logic of quantum mechanics' ([6]) or 'quantum logic', when 'quantum logic' is used in a sense more general than a kind of non-classical logic.

The consideration behind MFQT is the following: Roughly speaking, quantum theory was first built by manipulating some sophisticated mathematical objects, like complex-valued functions, so that the numbers coming out of calculations matched those from experiments. Then von Neumann summarized it into a theory in which the mathematical structures called Hilbert spaces over $\mathbb{C}^{3}$ are used in a particular way ${ }^{4}$ as mathematical models of quantum systems ([36]). This is called the Hilbert space formalism of quantum theory and has become the standard form of quantum theory $([21,33])$. However, all of this is developed with more mathematical skills than physical intuitions. The mathematical model of a quantum system, a Hilbert space over $\mathbb{C}$, has complicated algebraic, geometric and topological structure. Hence the contents of this theory are hard to discover, and, even if discovered, are hard to apprehend. In my understanding, the basic question of MFQT can be asked from two different but complementary perspectives. From the perspective of mathematics, the question is why Hilbert spaces over $\mathbb{C}$ are used in such a particular way as mathematical models of quantum systems. From the perspective of physics, the question is what are the characteristics in quantum physics of some basic physical concepts, which force us to use mathematical models different from those in classical physics.

To answer this question, the paradigm of research in MFQT mainly consists of four steps: First, choose and start from some basic concepts in physics. This guarantees that the study is transparent in physics and will not be submerged by mathematical details. Second, find axioms, as simple and natural as possible, to characterize the features of these concepts in quantum theory. This helps us to understand these concepts, but at this step we do not know how much we

\footnotetext{
${ }^{1}$ The term '(testable) property' in quantum logic means the physical concept represented by subspaces of Hilbert spaces over $\mathbb{C}$ according to quantum theory. It is called 'experimental proposition' in [10].

${ }^{2}$ This acronym is not common in the literature. It may only be used in this paper, because the full term is a bit long.

$3 \mathbb{C}$ denotes the field of complex numbers.

${ }^{4}$ The way how a Hilbert space is used to model a quantum system is worth attention. The reason is that differential manifolds in classical physics and Hilbert spaces in quantum physics can both be considered as generalizations or enrichments of vector spaces. However, in classical physics each state is represented by a unique vector, while in quantum physics a state can be represented by two different vectors.
} 
have understood and whether our understanding is complete. Third, use simple ${ }^{5}$ mathematical structures to model these concepts. This sets the stage for the following last step. Fourth, prove representation theorems of the mathematical structures satisfying the axioms via Hilbert spaces over $\mathbb{C}$. A theorem of this kind will separate the simple mathematical structures into two classes, ones that can be abstracted from Hilbert spaces and ones that can not. Once such a theorem is proved, we learn that the axioms obtained in the second step form a complete description of the quantum features of the concepts. Note that it is not the case that every approach in MFQT consists of all of the above four steps. Especially, the fourth step may involve very hard mathematical problems that take years to solve.

The works of Mackey, Piron, Solèr, Holland, etc. collectively and typically exemplify this paradigm. The starting point is the concept of a property in physics. Then axioms governing conjunction, negation, etc. of properties are found in quantum theory. Lattices are the mathematical structures used to model the structure formed by properties of quantum systems, and nowadays lattices satisfying these axioms are usually called Piron lattices. Finally, Piron's Theorem ([32]) is a representation theorem of Piron lattices via generalized Hilbert spaces, which are a kind of mild algebraic generalizations of Hilbert spaces over $\mathbb{C}$. In further research $([24,27])$, lattice-theoretic conditions are found which finally characterize the lattices that can be abstracted from Hilbert spaces over $\mathbb{C}$.

The approach mentioned above is the first and among the most successful in MFQT. From my point of view, in the literature, there are many other works that could be considered of this kind. For example, algebras are used to model the structure formed by effects of quantum systems ([12]); Kripke frames ([17]), labelled transition systems ([5]) and probabilistic transition systems ([28]) are used to model different aspects of the structure formed by the (pure) states of quantum systems. If I may, the work of categorical quantum mechanics $([1,13])$ can be considered as using categories to model compositions of quantum systems, and the work in the book [14] as reconstructing quantum theory from the concept of information.

It is worth mentioning that MFQT is related to Hilbert's Sixth Problem, i.e. mathematical treatment of the axioms of physics. Quantum theory is the guide instead of the opponent or rival of MFQT, and thus, as models of quantum systems, many mathematical structures in MFQT are not as sophisticated as Hilbert spaces. However, an advantage of the mathematical structures in MFQT is that they are tailored to highlight the features in quantum theory of the chosen physical concepts so that such features are not obscured by mathematical complexity. This is similar to the case that in synthetic geometry the axioms, the theorems and their proofs can be plainly and directly about important geometric objects like points and lines, while in analytic geometry the study of these geometric objects always has to be carried out by calculating numbers. In some sense, each approach in MFQT axiomatizes a part of quantum theory sectioned from the perspective of some physical concepts. In a word, MFQT can be considered as exemplifying a formal method in analysing concepts in theories, and thus is very helpful for the conceptual understanding of quantum

\footnotetext{
${ }^{5}$ Here the word 'simple' is used in an intuitive sense and mainly concerns the number and the complexity of primitives.
} 
theory.

As a by-product, MFQT may provide ways to discuss about quantum theory which are simple but still mathematically rigorous. This is because, to model and study some features of quantum theory, some simple mathematical structures may be sufficient according to MFQT. Moreover, studying the logics of the simple mathematical structures in MFQT may lead to sound, maybe even automatic, methods of reasoning about some quantum phenomena. Finally, most concepts focused on in MFQT are quite generic, and they are used in many other fields. The abstract study of quantum theory in MFQT facilitates finding applications of the Hilbert space formalism outside physics. This will deepen our understanding of this formalism and, in turn, quantum theory for which this formalism is originally devised.

\subsection{Background of this Paper}

The work in this paper belongs to MFQT. The starting concept is that of a (pure) state, which is widely used in the theoretical description of quantum systems as well as classical systems and computational systems. We will study the structure formed by the states of a quantum system under just one binary relation. This binary relation is such that, for any two states $s$ and $t$ of a quantum system, the relation holds for the pair $(s, t)$, if and only if they can be (perfectly) discriminated ${ }^{6}$ by one measurement of some observable of the system. To be precise,

the relation holds for the pair $(s, t)$, if and only if there is an observable of the system and two possible values $i$ and $j$ of the observable such that, if we know that the system is in either the state $s$ or the state $t$, then after a measurement of this observable we will know which is the case: the state is $s$ if and only if the outcome is $i$, and the state is $t$ if and only if the outcome is $j$.

Mathematically speaking, in the Hilbert space formalism of quantum theory, two states having this relation are called orthogonal.

The idea of this approach is not new at all. The orthogonality relation has been playing an important role in the proofs of theorems about Hilbert spaces and in the study of quantum physics. Since the 1970s, with the development of relational semantics for intentional logic, many researchers paid attention to the connection between the orthogonality relation and lattices arising in the study of quantum logic. Typical examples are [15, 17, 18, 19, 23, 31]. However, most of these works appear to be motivated by interest in lattice theory or logic instead of physics, though possibly [31] is an exception.

The work of this paper is a continuation of my works [41, 42]. In [41] a mathematical model of the orthogonality relation called a quantum Kripke frame is proposed; and, a duality, between a category formed by Piron lattices and one formed by quantum Kripke frames, is presented as a formalization of the state-property duality in quantum theory. In [42] quantum Kripke frames are studied from the perspective of geometry and proved to be able to model the projective geometry formed by the states of a quantum system. These

\footnotetext{
${ }^{6}$ There are different notions of discrimination between states in physics. For example, two are discussed in [39], and one of them called perfect discrimination is the one used here.
} 
results suggest that quantum Kripke frames could be good qualitative models of quantum systems. It is worth noting that quantum Kripke frames are defined by five conditions on a binary relation which are simpler than most conditions discussed in [23].

A main difference between this paper and the literature is that here the conceptual and physical significance, instead of mathematical properties, of quantum Kripke frames is the focus and is studied in two ways. On the one hand, I prove a mathematical theorem (Theorem 2.13) which makes precise and explicit the close connection between quantum Kripke frames and Hilbert spaces. On the other hand, I reflect on the definition of quantum Kripke frames and give physical hypotheses that support it. This brings some interesting insights into quantum theory and physical theories in general. Our conclusion is that just a few simple conditions satisfied by the orthogonality relation turn out to determine a large proportion of the structure formed by the states that is physically significant, and thus quantum Kripke frames are able to model a surprisingly large qualitative part of quantum theory. ${ }^{7}$

The rest of this paper is organized as follows. In Section 2 I will abstract the notion of quantum Kripke frames from quantum theory and prove the theorem (Theorem 2.13) that relates quantum Kripke frames to Hilbert spaces. Section 3 is an intermezzo, which tackles some technical issues so that the following section goes more fluently. In Section 4, I will give six hypotheses in physics which reveal the rationale behind the definition of quantum Kripke frames. Especially, I will reveal an implicit assumption in theories of classical and quantum physics (Remark 4.1), and I will prove a theorem (Proposition 4.8) which formalizes the idea that the Superposition Principle is sufficient for explaining all differences between quantum theory and classical physics. Moreover, I will present a qualitative version of quantum theory in which quantum Kripke frames are used as mathematical models of quantum systems. This highlights some qualitative features of measurements in quantum theory. Section 5 contains the conclusion and some discussion about future work. Finally, in the Appendix I develop the technicalities used in the proof of Proposition 4.5 in Section 4.

\section{Quantum Kripke Frame}

\subsection{The Non-Orthogonality Relation}

According to quantum theory, a quantum system is described by a Hilbert space $^{8} \mathcal{H}$ over $\mathbb{C}$ in such a way that the states of the system are modelled by the one-dimensional subspaces ${ }^{9}$ of $\mathcal{H} .{ }^{10}$

Denote by $\Sigma_{\mathcal{H}}$ the set of one-dimensional subspaces of $\mathcal{H}$; and define a binary relation $\rightarrow_{\mathcal{H}}$ on $\Sigma_{\mathcal{H}}$, called the non-orthogonal relation, such that, for any $s, t \in$ $\Sigma_{\mathcal{H}}, s \rightarrow_{\mathcal{H}} t$, if and only if there are $\mathbf{s} \in s$ and $\mathbf{t} \in t$ such that $(\mathbf{s}, \mathbf{t}) \neq 0$.

\footnotetext{
${ }^{7}$ Note that this point is non-trivial. For example, if we start from the concept of mixed states, it is not clear from the literature that a theory with similar nice features can be built.

${ }^{8}$ In this paper, all vector spaces, including Hilbert spaces, are assumed not to be $\{\mathbf{0}\}$.

${ }^{9}$ In this paper, a subspace of $\mathcal{H}$ means a closed linear subspace, i.e. it forms a Hilbert space with the addition, the scalar multiplication and the inner product inherited from $\mathcal{H}$.

${ }^{10}$ In many textbooks, e.g. [33], it is usually written that the states of the system are modelled by unit vectors of $\mathcal{H}$. However, according to the measurement postulate, in predicting experimental results there is no difference between a unit vector $\mathbf{v}$ and a non-zero scalar multiplication $c \mathbf{v}$ of it. Hence the statement here is more precise.
} 
In the literature, it is more common to focus on the complement of $\rightarrow \mathcal{H}$ in $\Sigma_{\mathcal{H}} \times \Sigma_{\mathcal{H}}$, i.e. the orthogonality relation. Following [42] it is more convenient to focus on $\rightarrow \mathcal{H}$, which is reflexive.

Mathematical as the definition is, the non-orthogonality relation has a clear physical meaning. $s \rightarrow_{\mathcal{H}} t$ can be interpreted in two ways, which are equivalent according to quantum theory: on the one hand, $s$ and $t$ cannot be discriminated by one measurement of an observable, as is mentioned in Subsection 1.2; on the other hand, there is a measurement such that one performance of it could trigger the system to change from one state to the other.

The following definitions from [42] are abstracted from a structure of the form $\left(\Sigma_{\mathcal{H}}, \rightarrow_{\mathcal{H}}\right)$ :

Definition 2.1. A Kripke frame $\mathfrak{F}$ is an ordered pair $(\Sigma, \rightarrow)$ in which $\Sigma$ is a non-empty set and $\rightarrow$ is a binary relation on $\Sigma$.

Definition 2.2. Let $\mathfrak{F}=(\Sigma, \rightarrow)$ be a Kripke frame.

- $s \not \rightarrow t$ means that $s \rightarrow t$ does not hold.

- For each $P \subseteq \Sigma$, the orthocomplement of $P$, denoted by $\sim P$, is the set $\{s \in \Sigma \mid s \not \rightarrow t$, for each $t \in P\}$.

- $P \subseteq \Sigma$ is closed, if $P=\sim \sim P$.

- $\mathcal{L}_{\mathfrak{F}}$ denotes the set of closed subsets of $\Sigma$.

- $s, t \in \Sigma$ are indistinguishable with respect to $P \subseteq \Sigma$, denoted by $s \approx_{P} t$, if $s \rightarrow w \Leftrightarrow t \rightarrow w$ holds for each $w \in P$.

- For each $P \subseteq \Sigma$ and $s \in \Sigma, s^{\prime} \in \Sigma$ is called a representative of $s$ in $P$, if $s^{\prime} \in P$ and $s \approx_{P} s^{\prime}$.

Definition 2.3. A quantum Kripke frame is a Kripke frame $\mathfrak{F}=(\Sigma, \rightarrow)$ satisfying all of the following five conditions:

1. Reflexivity: for each $s \in \Sigma, s \rightarrow s$.

2. Symmetry: for any $s, t \in \Sigma$, if $s \rightarrow t$, then $t \rightarrow s$.

3. Separation: for any $s, t \in \Sigma$, if $s \neq t$, then there is a $w \in \Sigma$ such that $w \rightarrow s$ but $w \not \rightarrow t$.

4. Superposition: for any $s, t \in \Sigma$, there is a $w \in \Sigma$ such that $w \rightarrow s$ and $w \rightarrow t$.

5. Representation: for any $P \subseteq \Sigma$ and $s \in \Sigma$, if $P=\sim \sim P$ and $s \notin \sim P$, then there is a representative of $s$ in $P$.

Reflexivity and Symmetry are well-known conditions satisfied by the nonorthogonality relation, and their logical properties have been studied in [15, 18, 19], etc. Separation is from [31]. It intuitively means that, for any two distinct states, there is always a state which can distinguish between, and in this sense separate, them by being non-orthogonal to one of them but not the other. Superposition is from [5]. It is a manifestation of the famous Superposition Principle in quantum theory. Representation is the counterpart of the Orthogonal Decomposition Theorem at the level of one-dimensional subspaces. 
Now we show that quantum Kripke frames are indeed abstracted from Hilbert spaces by mathematical results.

Proposition 2.4. For each Hilbert space $\mathcal{H}$ over $\mathbb{C}, \mathfrak{F}_{\mathcal{H}}=\left(\Sigma_{\mathcal{H}}, \rightarrow_{\mathcal{H}}\right)$ satisfies Reflexivity, Symmetry, Separation and Superposition.

Proof. Reflexivity and Symmetry follow from definite positiveness and conjugate symmetry of the inner product, respectively.

For Separation, let $s, t \in \Sigma_{\mathcal{H}}$ be arbitrary. Assume that $s \neq t$. Take $\mathbf{s} \in$ $s \backslash\{\mathbf{0}\}$ and $\mathbf{t} \in t \backslash\{\mathbf{0}\}$. Since $s \neq t, \mathbf{s}$ and $\mathbf{t}$ are linearly independent, so $\mathbf{w}=\mathbf{s}-\frac{(\mathbf{t}, \mathbf{s})}{(\mathbf{t}, \mathbf{t})} \mathbf{t} \neq \mathbf{0}$. Consider the one-dimensional subspace $w$ generated by $\mathbf{w}$. In fact, this is the orthogonal projection of the subspace $s$ on the orthocomplement of the subspace $t$. Note that

$$
\begin{aligned}
& (\mathbf{w}, \mathbf{s})=\left(\mathbf{s}-\frac{(\mathbf{t}, \mathbf{s})}{(\mathbf{t}, \mathbf{t})} \mathbf{t}, \mathbf{s}\right)=\frac{(\mathbf{s}, \mathbf{s})(\mathbf{t}, \mathbf{t})-(\mathbf{s}, \mathbf{t})(\mathbf{t}, \mathbf{s})}{(\mathbf{t}, \mathbf{t})} \\
& (\mathbf{w}, \mathbf{t})=\left(\mathbf{s}-\frac{(\mathbf{t}, \mathbf{s})}{(\mathbf{t}, \mathbf{t})} \mathbf{t}, \mathbf{t}\right)=\frac{(\mathbf{s}, \mathbf{t})(\mathbf{t}, \mathbf{t})-(\mathbf{s}, \mathbf{t})(\mathbf{t}, \mathbf{t})}{(\mathbf{t}, \mathbf{t})}=0
\end{aligned}
$$

On the one hand, because $\mathbf{s}$ and $\mathbf{t}$ are linearly independent, by Cauchy-Schwarz inequality $(\mathbf{s}, \mathbf{s})(\mathbf{t}, \mathbf{t})-(\mathbf{s}, \mathbf{t})(\mathbf{t}, \mathbf{s})>0$, so $w \rightarrow_{\mathcal{H}} s$. On the other hand, for any $\mathbf{w}^{\prime} \in w$ and $\mathbf{t}^{\prime} \in t$, there are $a, b \in \mathbb{C}$ such that $\mathbf{w}^{\prime}=a \mathbf{w}$ and $\mathbf{t}^{\prime}=b \mathbf{t}$, so $\left(\mathbf{w}^{\prime}, \mathbf{t}^{\prime}\right)=(a \mathbf{w}, b \mathbf{t})=a^{*} b(\mathbf{w}, \mathbf{t})=0$. Hence $w \rightarrow_{\mathcal{H}} t$.

For Superposition, let $s, t \in \Sigma_{\mathcal{H}}$ be arbitrary. If $s \rightarrow_{\mathcal{H}} t$, let $w=s$, and thus $w \rightarrow_{\mathcal{H}} t$. Moreover, by Reflexivity $w \rightarrow_{\mathcal{H}} s$. If $s \rightarrow_{\mathcal{H}} t$, then take $\mathbf{s} \in s \backslash\{\mathbf{0}\}$ and $\mathbf{t} \in t \backslash\{\mathbf{0}\}$. Since $s \rightarrow_{\mathcal{H}} t,(\mathbf{s}, \mathbf{t})=0$ and $(\mathbf{t}, \mathbf{s})=(\mathbf{s}, \mathbf{t})^{*}=0^{*}=0$. Hence

$$
(\mathbf{s}+\mathbf{t}, \mathbf{s}+\mathbf{t})=(\mathbf{s}, \mathbf{s})+(\mathbf{s}, \mathbf{t})+(\mathbf{t}, \mathbf{s})+(\mathbf{t}, \mathbf{t})=(\mathbf{s}, \mathbf{s})+(\mathbf{t}, \mathbf{t})>0
$$

By definite positiveness of the inner product $\mathbf{s}+\mathbf{t} \neq \mathbf{0}$. Consider the onedimensional subspace $w$ generated by $\mathbf{s}+\mathbf{t}$. Note that

$$
\begin{aligned}
& (\mathbf{s}+\mathbf{t}, \mathbf{s})=(\mathbf{s}, \mathbf{s})+(\mathbf{t}, \mathbf{s})=(\mathbf{s}, \mathbf{s})>0 \\
& (\mathbf{s}+\mathbf{t}, \mathbf{t})=(\mathbf{s}, \mathbf{t})+(\mathbf{t}, \mathbf{t})=(\mathbf{t}, \mathbf{t})>0
\end{aligned}
$$

Therefore, by definition $w \rightarrow_{\mathcal{H}} s$ and $w \rightarrow_{\mathcal{H}} t$.

To prove Representation we introduce a simple lemma.

Lemma 2.5. Let $\mathcal{H}$ be a Hilbert space over $\mathbb{C}$. For each $P \subseteq \Sigma_{\mathcal{H}}$, if $P \neq \emptyset$ and $P=\sim_{\mathcal{H}} \sim_{\mathcal{H}} P$, then $\bigcup P$ is a subspace of $\mathcal{H}$.

Proof. By Theorem 3 in Section 6 of Chapter III in [8] (p.71) $(\bigcup P)^{\perp \perp}$ is a subspace of $\mathcal{H}$, where, for each $W \subseteq \mathcal{H}$,

$$
W^{\perp} \stackrel{\text { def }}{=}\{\mathbf{s} \in \mathcal{H} \mid(\mathbf{s}, \mathbf{t})=0 \text { holds for each } \mathbf{t} \in W\}
$$

Hence, to show that $\bigcup P$ is a subspace of $\mathcal{H}$, it suffices to show that $\bigcup P=$ $(\bigcup P)^{\perp \perp}$. In the following, we prove this.

First, $\bigcup P \subseteq(\bigcup P)^{\perp \perp}$ follows from the definition of $(\cdot)^{\perp}$.

Second, let $\mathbf{s} \in(\bigcup P)^{\perp \perp}$ be arbitrary. If $\mathbf{s}=\mathbf{0}$, since $P \neq \emptyset$, we have $\mathbf{s} \in \bigcup P$. If $\mathbf{s} \neq \mathbf{0}$, denote by $s$ the one-dimensional subspace generated by $\mathbf{s}$. 
Let $t \in \sim_{\mathcal{H}} P$ be arbitrary. It follows that $\mathbf{t} \in(\bigcup P)^{\perp}$ holds for each $\mathbf{t} \in t$. Since $\mathbf{s} \in(\bigcup P)^{\perp \perp},(\mathbf{s}, \mathbf{t})=0$ holds for each $\mathbf{t} \in t$. It follows that $s A_{\mathcal{H}} t$. Since $t$ is arbitrary, $s \in \sim_{\mathcal{H}} \sim_{\mathcal{H}} P=P$, and thus $\mathbf{s} \in s \subseteq \bigcup P$. Therefore, $(\bigcup P)^{\perp \perp} \subseteq \bigcup P$.

Proposition 2.6. Let $\mathcal{H}$ be a Hilbert space over $\mathbb{C}$. $\left(\Sigma_{\mathcal{H}}, \rightarrow_{\mathcal{H}}\right)$ satisfies Representation.

Proof. Assume that $P \subseteq \Sigma_{\mathcal{H}}$ and $s \in \Sigma_{\mathcal{H}}$ satisfy $P=\sim_{\mathcal{H}} \sim_{\mathcal{H}} P$ and $s \notin \sim_{\mathcal{H}} P$. Note that $P \neq \emptyset$; otherwise, it can be shown that $s \notin \sim_{\mathcal{H}} P=\sim_{\mathcal{H}} \emptyset=\Sigma_{\mathcal{H}}$, contradicting $s \in \Sigma_{\mathcal{H}}$. Since $P=\sim_{\mathcal{H}} \sim_{\mathcal{H}} P$, by the previous lemma $\bigcup P$ is a subspace of $\mathcal{H}$. Take an $\mathbf{s} \in s \backslash\{\mathbf{0}\}$. By the Orthogonal Decomposition Theorem (see e.g. Theorem 3.6.2 in [8]) there are $\mathbf{s}_{\|} \in \bigcup P$ and $\mathbf{s}_{\perp} \in(\bigcup P)^{\perp}$ such that $\mathbf{s}=\mathbf{s}_{\|}+\mathbf{s}_{\perp}$. Note that $\mathbf{s}_{\|} \neq \mathbf{0}$; otherwise $\mathbf{s}=\mathbf{s}_{\perp}$, and it follows that $s \in \sim_{\mathcal{H}} P$, contradicting the assumption. Denote by $s_{\|}$the one-dimensional subspace generated by $\mathbf{s}_{\|}$. Since $\mathbf{s}_{\|} \in \bigcup P, s_{\|} \in P$. Moreover, for any $\mathbf{t} \in \bigcup P$, $(\mathbf{s}, \mathbf{t})=\left(\mathbf{s}_{\|}, \mathbf{t}\right)$. Hence, for each $t \in P, s \rightarrow_{\mathcal{H}} t$ if and only if $s_{\|} \rightarrow_{\mathcal{H}} t$.

As a result, we have the following:

Proposition 2.7. For each Hilbert space $\mathcal{H}$ over $\mathbb{C}, \mathfrak{F}_{\mathcal{H}}=\left(\Sigma_{\mathcal{H}}, \rightarrow_{\mathcal{H}}\right)$ is a quantum Kripke frame, called the quantum Kripke frame induced by $\mathcal{H}$.

We end this subsection by a simple but useful lemma.

Lemma 2.8. Let $\mathfrak{F}=(\Sigma, \rightarrow)$ be a quantum Kripke frame.

1. $\sim \emptyset=\Sigma$ and $\sim \Sigma=\emptyset$, and thus $\sim \emptyset=\emptyset$ and $\sim \Sigma=\Sigma$, i.e. $\emptyset, \Sigma \in \mathcal{L}_{\mathfrak{F}}$.

2. For any $P, Q \subseteq \Sigma, P \subseteq Q$ implies that $\sim Q \subseteq \sim P$.

3. For each $P \subseteq \Sigma, P \subseteq \sim \sim P$.

4. For each $P \subseteq \Sigma, \sim P$ is closed.

5. $\bigcap_{i \in I} P_{i} \in \mathcal{L}_{\mathfrak{F}}$, if $P_{i} \in \mathcal{L}_{\mathfrak{F}}$ holds for each $i \in I$.

6. For each $s \in \Sigma,\{s\}$ is closed.

7. For any $P \subseteq \Sigma$ and $s, t, t^{\prime} \in \Sigma$, if $P$ is closed and both $t$ and $t^{\prime}$ are representatives of $s$ in $P$, then $t=t^{\prime}$.

Proof. Items 1 to 6 are Lemma 3.6 in [42] and Lemma 8 in [41].

For Item 7, assume that both $t$ and $t^{\prime}$ are representatives of $s$ in $P$. Suppose (towards a contradiction) that $t \neq t^{\prime}$. By Separation there is a $w \in \Sigma$ such that $w \rightarrow t$ and $w \nrightarrow t^{\prime}$. Since $t$ is a representative of $s$ in $P, t \in P$. From $w \rightarrow t$ and $t \in P$ we know that $w \notin \sim P$. Since $P$ is closed, by Representation there is a representative $w^{\prime}$ of $w$ in $P$. On the one hand, since $w \rightarrow t$ and $t \in P, w^{\prime} \rightarrow t$. Since $w^{\prime} \in P, s \rightarrow w^{\prime}$. On the other hand, since $w \nrightarrow \rightarrow t^{\prime}$ and $t^{\prime} \in P, w^{\prime} \nrightarrow \rightarrow t^{\prime}$. Since $w^{\prime} \in P, s \nrightarrow \rightarrow w^{\prime}$. We have got a contradiction. 


\subsection{Quantum Kripke Frames and Hilbert Spaces}

In this subsection we study the connection between quantum Kripke frames and Hilbert spaces via some representation theorems between quantum Kripke frames and special kinds of vector spaces. It will shed light on how abstract quantum Kripke frames are, compared to Hilbert spaces.

We start from briefly reviewing the definition of generalized Hilbert spaces $([32,35])$, which are mild algebraic generalizations of Hilbert spaces over $\mathbb{C}$.

Definition 2.9. A division ring is a ring in which every non-zero element has a multiplicative inverse (and the multiplication may not be commutative.)

An involution on a division $\operatorname{ring} \mathcal{K}=(K,+, \cdot, 0,1)$ is a function $\mu: K \rightarrow K$ satisfying all of the following:

1. $\mu$ is bijective;

2. $\mu(x+y)=\mu(x)+\mu(y)$ and $\mu(x \cdot y)=\mu(y) \cdot \mu(x)$, for any $x, y \in K$;

3. $(\mu \circ \mu)(x)=x$, for every $x \in K$;

An Hermitian form on a vector space $V$ over a division $\operatorname{ring} \mathcal{K}=(K,+, \cdot, 0,1)$ is a function $\Phi: V \times V \rightarrow K$ satisfying all of the following:

1. $\Phi(\mathbf{u}+\mathbf{v}, \mathbf{w})=\Phi(\mathbf{u}, \mathbf{w})+\Phi(\mathbf{v}, \mathbf{w})$, for any $\mathbf{u}, \mathbf{v}, \mathbf{w} \in V$;

2. $\Phi(x \mathbf{v}, \mathbf{w})=x \cdot \Phi(\mathbf{v}, \mathbf{w})$, for any $\mathbf{v}, \mathbf{w} \in V$ and $x \in K$;

3. there is an involution $\mu$ on $\mathcal{K}$ such that $\Phi(\mathbf{v}, \mathbf{w})=\mu(\Phi(\mathbf{w}, \mathbf{v}))$ holds for any $\mathbf{v}, \mathbf{w} \in V$.

$\mu$ is called the accompanying involution of $\Phi$.

A generalized Hilbert space is a vector space $V$ over some division ring $\mathcal{K}$ equipped with an Hermitian form $\Phi$ satisfying the following condition:

(*) for every $E \subseteq V$, if $E=\left(E^{\perp}\right)^{\perp}$, then $V=\left\{\mathbf{u}+\mathbf{v} \mid \mathbf{u} \in E\right.$ and $\left.\mathbf{v} \in E^{\perp}\right\}$;

where $E^{\perp} \stackrel{\text { def }}{=}\{\mathbf{u} \in V \mid \Phi(\mathbf{u}, \mathbf{v})=0$ holds for each $\mathbf{v} \in E\}$.

In this definition, the division ring, the involution and the Hermitian form are generalizations of the field of complex numbers, the complex conjugate and the inner product, respectively. The condition $(*)$, which is purely algebraic and is equivalent to the Orthogonal Decomposition Theorem in a Hilbert space, replaces the condition of metric completeness.

An idea from Amemiya and Araki shows that a generalized Hilbert space $V$ is a Hilbert space over $\mathbb{C}$, if its underlying division ring is $\mathbb{C}$ and the accompanying involution of the Hermitian form is the complex conjugate. In this situation, the Hermitian form with which $V$ is equipped is an inner product by definition $^{11}$, so $V$ is a pre-Hilbert space, i.e. a vector space over $\mathbb{C}$ equipped with an inner product. Then the result of Step 1 in the proof of the Theorem in [4] (p.425) is given plainly by the condition $(*)$ above, where in [4] it follows from orthomodularity of $L(V)$. The remaining steps in the proof of the Theorem in [4] (pp.426-427) shows that $V$ is complete and thus is a Hilbert space.

We cite a result in the literature and use it to prove the representation theorem of quantum Kripke frames via generalized Hilbert spaces.

\footnotetext{
${ }^{11}$ In fact, there is a minor conventional difference: an inner product is conjugate on the first argument, while an Hermitian form is conjugate on the second argument.
} 
Theorem 2.10.

1. For every quantum Kripke frame $\mathfrak{F}=(\Sigma, \rightarrow), \mathbf{G}(\mathfrak{F})=(\Sigma, \sim \sim\{\cdot, \cdot\}, \not \rightarrow)$ is an irreducible Hilbertian geometry ${ }^{12}$.

2. For every irreducible Hilbertian geometry $\mathfrak{G}=(G, \star, \perp), \mathbf{F}(\mathfrak{G})=(G, \not \chi)$ is a quantum Kripke frame.

3. $\mathbf{G}$ is a class function ${ }^{13}$ from the class of quantum Kripke frames to that of irreducible Hilbertian geometries.

$\mathbf{F}$ is a class function from the class of irreducible Hilbertian geometries to that of quantum Kripke frames.

For any quantum Kripke frame $\mathfrak{F}$ and irreducible Hilbertian geometry $\mathfrak{G}$,

$$
(\mathbf{F} \circ \mathbf{G})(\mathfrak{F})=\mathfrak{F} \quad(\mathbf{G} \circ \mathbf{F})(\mathfrak{G})=\mathfrak{G}
$$

Proof. It follows from Theorem 4.22 in [42].

Theorem 2.11. For each Kripke frame $\mathfrak{F}=(\Sigma, \rightarrow)$, the following are equivalent:

(i) $\mathfrak{F}$ is a quantum Kripke frame, and there are $\left\{s_{1}, s_{2}, s_{3}, s_{4}\right\} \in \Sigma$ such that $s_{i} \not \rightarrow s_{j}$ for any distinct $i, j \in\{1,2,3,4\}$;

(ii) there is a generalized Hilbert space $V$ of dimension at least 4 such that $\mathfrak{F} \cong \mathfrak{F}_{V}$, where $\mathfrak{F}_{V}=\left(\Sigma_{V}, \rightarrow_{V}\right)$ is such that

1. $\Sigma_{V}$ is the set of all one-dimensional subspaces of $V$;

2. for any $s, t \in \Sigma_{V}, s \rightarrow_{V} t$, if $\Phi(\mathbf{s}, \mathbf{t}) \neq 0$ for some $\mathbf{s} \in s$ and $\mathbf{t} \in t$; here $\Phi$ is the Hermitian form of $V$.

Moreover, if they exist, both $V$ and its underlying division ring are unique up to isomorphism, and $\Phi$ is unique up to a constant multiple.

Proof. From (ii) to (i): The proof of that $\mathfrak{F}_{V}$ is a quantum Kripke frame is similar to that of Proposition 2.7, noting that the latter only uses the Orthogonal Decomposition Theorem instead of the full power of metric completeness.

Since $V$ is of dimension at least 4, by the Gram-Schmidt process we can get four pairwise orthogonal elements in $\Sigma_{V}$.

Since $\mathfrak{F} \cong \mathfrak{F}_{V}$, (i) holds.

From (i) to (ii): Since $\mathfrak{F}$ is a quantum Kripke frame, by Theorem 2.10 $\mathbf{G}(\mathfrak{F})=(\Sigma, \sim \sim\{\cdot, \cdot\}, \not \rightarrow)$ is an irreducible Hilbertian geometry. Since there are four pairwise orthogonal elements in $\Sigma$, it can be shown that $\mathbf{G}(\mathfrak{F})$ is of dimension at least 3. By Theorem 8.4.6 in [16] or Theorem 2.7.1 in [9] $\mathbf{G}(\mathfrak{F})$ is arguesian. By Theorem 82 in $[35]^{14}$ there is a generalized Hilbert space $V$ such that $\left(\Sigma_{V}, \star_{V}, \perp_{V}\right) \cong(\Sigma, \sim \sim\{\cdot, \cdot\}, \not \rightarrow)=\mathbf{G}(\mathfrak{F})$. Therefore, $\mathfrak{F}=(\Sigma, \rightarrow) \cong$

\footnotetext{
${ }^{12}$ Here the term 'Hilbertian geometry' is used in the sense of Definition 14.5.4 in [16] and Definition 2.4 in [42].

${ }^{13}$ The term 'class function' emphasizes that $\mathbf{G}$ is defined on a proper class. Though it behaves like a function, $\mathbf{G}$ is not a set and thus is not a function in the sense of $\mathbf{Z F C}$.

${ }^{14}$ Note that what is called an 'arguesian irreducible Hilbertian geometry' in this paper is called an 'arguesian Hilbert geometry' in [35].
} 
$\left(\Sigma_{V}, \not_{V}\right)=\left(\Sigma_{V}, \rightarrow_{V}\right)=\mathfrak{F}_{V}$. Moreover, $V$ is of dimension at least 4 , for $\mathbf{G}(\mathfrak{F})$ is of dimension at least 3 .

Uniqueness: The uniqueness of $V$ and its underlying division ring follows from Proposition 9.4.4 in [16], which is a result about arguesian projective geometry in general. The uniqueness of $\Phi$ follows from Proposition 83 in [35].

The conceptual significance of this theorem is that the five conditions in the definition of quantum Kripke frames capture the essential properties of the non-orthogonality relation between states of quantum systems.

To make a step further, we can characterize quantum Kripke frames isomorphic to those induced by infinite-dimensional Hilbert spaces over $\mathbb{R}, \mathbb{C}$ or $\mathbb{H}^{15}$ using the following theorem:

Theorem 2.12 (Solèr's Theorem [34]). Let $V$ be a generalized Hilbert space with $\mathcal{K}$ as the underlying division ring and $\Phi$ as the Hermitian form. $V$ is isomorphic to a Hilbert space over $\mathbb{R}, \mathbb{C}$ or $\mathbb{H}$, if there is a non-zero element $k$ in $\mathcal{K}$ and a set $\left\{\mathbf{v}_{i} \mid i \in \mathbb{N}\right\} \subseteq V$ such that

$$
\text { for any } i, j \in \mathbb{N}, \Phi\left(\mathbf{v}_{i}, \mathbf{v}_{j}\right)= \begin{cases}k, & \text { if } i=j \\ 0, & \text { if } i \neq j\end{cases}
$$

The condition in this theorem has been studied in frameworks that are more abstract $([3,24])$. Here we use a modified version of Aert's Axiom of Plane Transitivity ([3]). This condition is in terms of the automorphisms on a quantum Kripke frame, which by Wigner's Theorem ([37]) correspond to unitary or antiunitary operators, if the quantum Kripke frame is induced by a Hilbert space over $\mathbb{C}$. According to this condition, quantum Kripke frames induced by Hilbert spaces are the highly symmetric ones.

Theorem 2.13. Let $\mathfrak{F}=(\Sigma, \rightarrow)$ be a Kripke frame. The following are equivalent:

(i) there is an infinite-dimensional Hilbert space $V$ over $\mathbb{R}, \mathbb{C}$ or $\mathbb{H}$ such that $\mathfrak{F} \cong \mathfrak{F}_{V}$

(ii) $\mathfrak{F}$ is a quantum Kripke frame such that: (a) there is a set $\left\{v_{i} \mid i \in \mathbb{N}\right\} \subseteq \Sigma$ such that $v_{i} \nrightarrow \rightarrow v_{j}$ holds for any distinct $i, j \in \mathbb{N}$; and (b) for any $s, t \in \Sigma$ with $s \nrightarrow t$, there is an automorphism $F$ of $\mathfrak{F}$ such that $F(s)=t, F(t)=s$ and $F$ restricted to $\sim\{s, t\}$ is the identity.

Proof. From (i) to (ii): Assume (i). Without loss of generality, we identify $\mathfrak{F}$ with $\mathfrak{F}_{V}$, where $V$ is the infinite-dimensional Hilbert space over $\mathbb{R}, \mathbb{C}$ or $\mathbb{H}$ and $\Phi$ is the Hermitian form of $V$. By Theorem $2.11 \mathfrak{F}_{V}$ is a quantum Kripke frame.

For (a), since $V$ is infinite-dimensional, from an orthonormal basis of $V$ we can find $\left\{\mathbf{v}_{i} \mid i \in \mathbb{N}\right\} \subseteq V \backslash\{\mathbf{0}\}$ such that, for any distinct $i, j \in \mathbb{N}, \Phi\left(\mathbf{v}_{i}, \mathbf{v}_{j}\right)=0$. For each $i \in \mathbb{N}$, let $v_{i}$ be the one-dimensional subspace generated by $\mathbf{v}_{i}$. It can be verified that $\left\{v_{i} \mid i \in \mathbb{N}\right\} \subseteq \Sigma_{V}$ has the required property.

For (b), let $s, t \in \Sigma_{V}$ be such that $s \not_{V} t$. Since $V$ is a Hilbert space over $\mathbb{R}$, $\mathbb{C}$ or $\mathbb{H}$, we can find $\mathbf{s} \in s$ and $\mathbf{t} \in t$ such that $\Phi(\mathbf{s}, \mathbf{t})=0, \Phi(\mathbf{s}, \mathbf{s})=\Phi(\mathbf{t}, \mathbf{t})=1$ and can extend the set $\{\mathbf{s}, \mathbf{t}\}$ to an orthonormal basis $B$ of $V$ by Theorem 2.2.10 in [25]. Let $\mathrm{f}$ be the bijection on $B$ such that $\mathrm{f}(\mathbf{s})=\mathbf{t}, \mathbf{f}(\mathbf{t})=\mathbf{s}$ and $\mathbf{f}$ maps each

\footnotetext{
${ }^{15} \mathbb{H}$ denotes the division ring of quaternions.
} 
of other elements in $B$ to itself. Following the idea in the proof of Theorem 3 in Section 16 of Chapter I in [22] (p.30), we can extend $\mathrm{f}$ to an automorphism $f$ on $V$ by mapping a vector $\mathbf{v}=\sum_{\mathbf{b} \in B}(\mathbf{b}, \mathbf{v}) \mathbf{b}$ to the vector $\sum_{\mathbf{b} \in B}(\mathbf{b}, \mathbf{v}) \mathbf{f}(\mathbf{b})$. Define that $F(s)=f[s]^{16}$ for each $s \in \Sigma_{V}$. It can be shown that $F$ is an automorphism of $\mathfrak{F}_{V}$ such that $F(s)=t, F(t)=s$ and $F$ restricted to $\sim_{V}\{s, t\}$ is the identity.

From (ii) to (i): Assume (ii). By Theorem $2.11 \mathfrak{F} \cong \mathfrak{F}_{V}$ for some generalized Hilbert space $V$ equipped with an Hermitian form $\Phi$. We make the following claim:

Claim: For any $\mathbf{s}, \mathbf{t} \in V \backslash\{\mathbf{0}\}, \Phi(\mathbf{s}, \mathbf{t})=0$ implies that there is an $x$ in $\mathcal{K}$ such that $\Phi(\mathbf{s}, \mathbf{s})=\Phi(x \mathbf{t}, x \mathbf{t})$.

This claim is crucial in the following sense. By (ii) there is a set $\left\{v_{i} \mid i \in\right.$ $\mathbb{N}\} \subseteq \Sigma_{V}$ such that $v_{i} \not_{V} v_{j}$ holds for any distinct $i, j \in \mathbb{N}$. We can find a $\mathbf{v}_{i}^{\prime} \in v_{i} \backslash\{\mathbf{0}\}$ for each $i \in \mathbb{N}$ such that $\Phi\left(\mathbf{v}_{i}^{\prime}, \mathbf{v}_{j}^{\prime}\right)=0$ holds for any distinct $i, j \in \mathbb{N}$. Moreover, for each $i \in \mathbb{N} \backslash\{0\}$, by the claim there is an $x_{i}$ in $\mathcal{K}$ such that $\Phi\left(\mathbf{v}_{0}^{\prime}, \mathbf{v}_{0}^{\prime}\right)=\Phi\left(x_{i} \mathbf{v}_{i}^{\prime}, x_{i} \mathbf{v}_{i}^{\prime}\right)$. Let $\mathbf{v}_{0}=\mathbf{v}_{0}^{\prime}$ and $\mathbf{v}_{i}=x_{i} \mathbf{v}_{i}^{\prime}$ for each $i \in \mathbb{N} \backslash\{0\}$. It follows that $\left\{\mathbf{v}_{i} \mid i \in \mathbb{N}\right\}$ satisfies (1) in Theorem 2.12, and thus $V$ is isomorphic to an infinite-dimensional Hilbert space over $\mathbb{R}, \mathbb{C}$ or $\mathbb{H}$, so (i) follows.

It remains to prove the claim. Let $\mathbf{s}, \mathbf{t} \in V \backslash\{\mathbf{0}\}$ be arbitrary such that $\Phi(\mathbf{s}, \mathbf{t})=0$. Denote by $s$ and $t$ the one-dimensional subspaces generated by $\mathbf{s}$ and $\mathbf{t}$, respectively. Then $s \not_{V} t$. By (ii) there is an automorphism $F$ of $\mathfrak{F}_{V}$ such that $F(s)=t, F(t)=s$ and $F$ restricted to $\sim_{V}\{s, t\}$ is the identity. Since $F$ is an automorphism, $\left(F, F^{-1}\right)$ is an adjunction between $\mathbf{G}\left(\mathfrak{F}_{V}\right)$ and itself (Definition 14.4.1 in [16]), and thus $F$ is a continuous homomorphism by Proposition 14.4.4 in [16]. Moreover, it is also an orthogonal morphism on $\mathbf{G}\left(\mathfrak{F}_{V}\right)$ (Definition 14.3.1 in [16]). By Proposition 13.5.3 and Theorem 14.3.4 in [16] there is a quasi-linear map $f$ on $V^{17}$ and a $k$ in $\mathcal{K}$ such that $F(s)=f[s]$ holds for each $s \in \Sigma_{V}$ and $\Phi(f(\mathbf{u}), f(\mathbf{v}))=\sigma(\Phi(\mathbf{u}, \mathbf{v}) \cdot k)$ holds for any $\mathbf{u}, \mathbf{v} \in V$.

It follows from (ii) that $V$ is infinite-dimensional. Hence there are linearly independent $\mathbf{u}, \mathbf{v} \in V \backslash\{\mathbf{0}\}$ such that $u, v \in \sim_{V}\{s, t\}$, where $u$ and $v$ are the one-dimensional subspaces generated by $\mathbf{u}$ and $\mathbf{v}$, respectively.

Note that there is a non-zero $z$ in $\mathcal{K}$ such that $\sigma(y)=z \cdot y \cdot z^{-1}$ holds for every $y$ in $\mathcal{K}$ : Since $F$ restricted to $\sim_{V}\{s, t\}$ is the identity, $f(\mathbf{w})$ is in the one-dimensional subspace generated by $\operatorname{id}(\mathbf{w})$, for every $\mathbf{w} \in L(\{\mathbf{u}, \mathbf{v}\}) \backslash\{\mathbf{0}\}$, where $L(\{\mathbf{u}, \mathbf{v}\})$ is the subspace generated by $\mathbf{u}$ and $\mathbf{v}$ and id is the identity map on $L(\{\mathbf{u}, \mathbf{v}\})$. Since $\mathbf{u}$ and $\mathbf{v}$ are linearly independent, $L(\{\mathbf{u}, \mathbf{v}\})$, as the image of id, is two-dimensional. By Lemma 6.3.4 in [16] there is a unique non-zero $z$ in $\mathcal{K}$ such that $f(\mathbf{w})=z \operatorname{id}(\mathbf{w})=z \mathbf{w}$ for every $\mathbf{w} \in L(\{\mathbf{u}, \mathbf{v}\})$. Now let $y$ in $\mathcal{K}$ be arbitrary. Consider $y \mathbf{u} \in L(\{\mathbf{u}, \mathbf{v}\})$. On the one hand, $f(y \mathbf{u})=\sigma(y) f(\mathbf{u})=(\sigma(y) \cdot z) \mathbf{u}$. On the other hand, $f(y \mathbf{u})=z(y \mathbf{u})=(z \cdot y) \mathbf{u}$. Therefore, $(\sigma(y) \cdot z) \mathbf{u}=(z \cdot y) \mathbf{u}$; and thus $\sigma(y)=z \cdot y \cdot z^{-1}$, for $z$ is non-zero.

Also note that $k=z^{*} \cdot z$, where $(\cdot)^{*}$ is the accompanying involution of $\Phi$ : On the one hand, $\Phi(f(\mathbf{u}), f(\mathbf{u}))=\sigma(\Phi(\mathbf{u}, \mathbf{u}) \cdot k)=z \cdot \Phi(\mathbf{u}, \mathbf{u}) \cdot k \cdot z^{-1}$. On the other hand, $\Phi(f(\mathbf{u}), f(\mathbf{u}))=\Phi(z \mathbf{u}, z \mathbf{u})=z \cdot \Phi(\mathbf{u}, \mathbf{u}) \cdot z^{*}$. Therefore, $z \cdot \Phi(\mathbf{u}, \mathbf{u}) \cdot z^{*}=z \cdot \Phi(\mathbf{u}, \mathbf{u}) \cdot k \cdot z^{-1}$, and so $k=z^{*} \cdot z$.

Now since $f[s]=F(s)=t$, there is a non-zero $l$ in $\mathcal{K}$ satisfying $f(\mathbf{s})=l \mathbf{t}$.

\footnotetext{
${ }^{16} f[s]$ is the image of $s$ under $f$. It makes sense, for $f$ is a function from $V$ to $V$ and $s \subseteq V$.

${ }^{17}$ This means that there is a division ring isomorphism $\sigma$ on $\mathcal{K}$ such that, for any $\mathbf{u}, \mathbf{v} \in V$ and $x$ in $\mathcal{K}, f(\mathbf{u}+\mathbf{v})=f(\mathbf{u})+f(\mathbf{v})$ and $f(x \mathbf{u})=\sigma(x) f(\mathbf{u})$. (Definition 6.6.10 in [16])
} 
Thus $\Phi(l \mathbf{t}, l \mathbf{t})=\Phi(f(\mathbf{s}), f(\mathbf{s}))=\sigma(\Phi(\mathbf{s}, \mathbf{s}) \cdot k)=z \cdot \Phi(\mathbf{s}, \mathbf{s}) \cdot z^{*} \cdot z \cdot z^{-1}=z \cdot \Phi(\mathbf{s}, \mathbf{s}) \cdot z^{*}$ Therefore, taking $x$ to be $z^{-1} \cdot l$, we have $\Phi(\mathbf{s}, \mathbf{s})=\Phi(x \mathbf{t}, x \mathbf{t})$.

Finally, note that $\mathbb{R}, \mathbb{C}$ and $\mathbb{H}$ can be distinguished by first-order conditions on division rings. Also note that every line in the projective geometry induced by a vector space $V$ (of dimension at least 3 ) over a division ring $\mathcal{K}$ can be made into a division ring isomorphic to $\mathcal{K}$ with addition and multiplication defined via geometric constructions. Therefore, quantum Kripke frames induced by Hilbert spaces over $\mathbb{R}, \mathbb{C}$ and $\mathbb{H}$ can be distinguished by first-order conditions on Kripke frames. However, although this idea is straightforward in mathematics, I have to confess that the physical significance of these first-order conditions is obscure. A promising alternative is to distinguish these three kinds of quantum Kripke frames by the structure formed by the automorphisms on a quantum Kripke frame, as is hinted by Mayet's paper [27].

\section{Technical Intermezzo: Opposite Family}

In this section, we introduce the notion of opposite families and prove some results about them, all of which will be used in Section 4 .

We fix a Kripke frame $\mathfrak{F}=(\Sigma, \rightarrow)$ satisfying Reflexivity and Symmetry throughout this section for convenience.

Note that Items 1 to 5 in Lemma 2.8 only need Reflexivity and Symmetry in their proofs, so they hold in $\mathfrak{F}$.

First, we give the definition of opposite families.

Definition 3.1. An opposite family in $\mathfrak{F}$ is a set $\left\{P_{i} \mid i \in I\right\} \subseteq \wp(\Sigma) \backslash\{\emptyset\}$ for some $I \neq \emptyset$ such that $s \not \rightarrow t$ holds for any $i, j \in I$ with $i \neq j, s \in P_{i}$ and $t \in P_{j}$.

An opposite family $\left\{P_{i} \mid i \in I\right\}$ in $\mathfrak{F}$ is maximal, if, for each opposite family $\left\{Q_{i} \mid i \in I\right\}$ in $\mathfrak{F}$, that $P_{i} \subseteq Q_{i}$ holds for each $i \in I$ implies that $P_{i}=Q_{i}$ holds for each $i \in I$.

Remark 3.2. Intuitively, an opposite family $\left\{P_{i} \mid i \in I\right\}$ is maximal, if no $P_{i}$ can be properly extended while keeping the resulting subset of $\wp(\Sigma) \backslash\{\emptyset\}$ being an opposite family.

When $I$ is a singleton, an opposite family $\left\{P_{i} \mid i \in I\right\}$ is maximal, if and only if $\left\{P_{i} \mid i \in I\right\}=\{\Sigma\}$.

Second, we prove a result relating opposite families and closed subsets.

Proposition 3.3. For each $P \subseteq \Sigma$, the following are equivalent:

(i) $P$ is closed;

(ii) there is a non-empty set $I$, a maximal opposite family $\left\{P_{i} \mid i \in I\right\}$ and a $j \in I$ such that $P=P_{j}$.

Moreover, for each closed $P \varsubsetneqq \Sigma,\{P, \sim P\}$ is the unique bipartite maximal opposite family in $\mathfrak{F}$ that contains $P$.

Proof. When $P=\Sigma$, the result follows from Remark 3.2 and Lemma 2.8. Hence it remains to prove the case when $P \neq \Sigma$. 
From (ii) to (i): Assume that (ii) holds. Define, for each $i \in I$,

$$
P_{i}^{\prime}= \begin{cases}\sim \sim P_{i}, & \text { if } i=j \\ P_{i}, & \text { if } i \neq j\end{cases}
$$

It can be verified that it is an opposite family. Since both $\left\{P_{i} \mid i \in I\right\}$ and $\left\{P_{i}^{\prime} \mid i \in I\right\}$ are opposite families, $P_{j} \subseteq \sim \sim P_{j}=P_{j}^{\prime}$ by Lemma 2.8 and $P_{i} \subseteq P_{i}^{\prime}$ holds for each $i \in I \backslash\{j\}$, we get $P_{j}=\sim \sim P_{j}$, because $\left\{P_{i} \mid i \in I\right\}$ is maximal.

From (i) to (ii): By definition $\{P, \sim P\}$ is an opposite family.

To prove that $\{P, \sim P\}$ is maximal, assume that $P^{\prime} \subseteq \Sigma$ and $Q^{\prime} \subseteq \Sigma$ are such that $P \subseteq P^{\prime}, \sim P \subseteq Q^{\prime}$ and $\left\{P^{\prime}, Q^{\prime}\right\}$ is an opposite family in $\mathfrak{F}$. Combining this assumption with Lemma 2.8 and (i), we have

$$
P^{\prime} \subseteq \sim Q^{\prime} \subseteq \sim \sim P=P \subseteq P^{\prime} \quad \text { and } \quad Q^{\prime} \subseteq \sim P^{\prime} \subseteq \sim P \subseteq Q^{\prime}
$$

Therefore, $P=P^{\prime}$ and $\sim P=Q^{\prime}$, so $\{P, \sim P\}$ is maximal.

For Uniqueness: Let $P \varsubsetneqq \Sigma$ be closed. Assume that $\{P, Q\}$ is a maximal opposite family. By definition $Q \subseteq \sim P$ and $P \subseteq P$. Since $\{P, \sim P\}$ is an opposite family, by the assumption $Q=\sim P$, and thus $\{P, Q\}=\{P, \sim P\}$.

Third, we prove a useful characterization of maximal opposite families.

Lemma 3.4. Let $\left\{P_{i} \mid i \in I\right\}$ be an opposite family in $\mathfrak{F}$. The following are equivalent:

(i) $\left\{P_{i} \mid i \in I\right\}$ is maximal;

(ii) for each $i^{*} \in I, P_{i^{*}}=\bigcap\left\{\sim P_{i} \mid i \in I \backslash\left\{i^{*}\right\}\right\}$.

Proof. If $I$ is a singleton, the result holds by Remark 3.2, where the convention $\bigcap\left\{Q_{j} \mid j \in \emptyset\right\}=\Sigma$ is used. If $I$ is not a singleton, the proof goes as follows:

From (i) to (ii): Let $i^{*} \in I$ be arbitrary. Define, for each $i \in I$,

$$
Q_{i}= \begin{cases}\bigcap_{1}\left\{\sim P_{i} \mid i \in I \backslash\left\{i^{*}\right\}\right\}, & \text { if } i=i^{*} \\ P_{i}, & \text { if } i \neq i^{*}\end{cases}
$$

It can be verified to be an opposite family, for $\left\{P_{i} \mid i \in I\right\}$ is an opposite family. Moreover, by definition $P_{i} \subseteq Q_{i}$ holds for each $i \in I \backslash\left\{i^{*}\right\}$. For $i^{*}$, because $P_{i^{*}} \subseteq \sim P_{i}$ holds for each $i \in I \backslash\left\{i^{*}\right\}, P_{i^{*}} \subseteq \bigcap\left\{\sim P_{i} \mid i \in I \backslash\left\{i^{*}\right\}\right\}=Q_{i^{*}}$. By (i) $P_{i^{*}}=Q_{i^{*}}=\bigcap\left\{\sim P_{i} \mid i \in I \backslash\left\{i^{*}\right\}\right\}$.

From (ii) to (i): Assume that $\left\{Q_{i} \mid i \in I\right\}$ is an opposite family such that $P_{i} \subseteq Q_{i}$ holds for each $i \in I$. Let $i^{*} \in I$ be arbitrary. Since $\left\{Q_{i} \mid i \in I\right\}$ is an opposite family, by definition $Q_{i^{*}} \subseteq \bigcap\left\{\sim Q_{i} \mid i \in I \backslash\left\{i^{*}\right\}\right\}$. Moreover, since $P_{i} \subseteq Q_{i}$ holds for each $i \in I, \bigcap\left\{\sim Q_{i} \mid i \in I \backslash\left\{i^{*}\right\}\right\} \subseteq \bigcap\left\{\sim P_{i} \mid i \in I \backslash\left\{i^{*}\right\}\right\}$. As a result, $Q_{i^{*}} \subseteq \bigcap\left\{\sim Q_{i} \mid i \in I \backslash\left\{i^{*}\right\}\right\} \subseteq \bigcap\left\{\sim P_{i} \mid i \in I \backslash\left\{i^{*}\right\}\right\}$. By (ii) $Q_{i^{*}} \subseteq P_{i^{*}}$, and thus $P_{i^{*}}=Q_{i^{*}}$. Since $i^{*} \in I$ is arbitrary, (i) holds.

\section{Quantum Kripke Frames as Models for Quan- tum Systems}

In Section 2, starting from the standard models for quantum systems, we define quantum Kripke frames and discuss how they relate to Hilbert spaces. 
In this section, we will study the rationale behind the definition of quantum Kripke frames in the form of six intuitive and physically significant hypotheses about the indiscriminability-by-one-measurement relation. From this we see how a quantum Kripke frame can be a qualitative model for a quantum system and what insight about quantum theory we can get from the perspective of states and the indiscriminability relation between states.

\subsection{Discussion on Reflexivity and Symmetry}

In the context of this paper, the intuition behind the abstract relation in a Kripke frame is the indiscriminability-by-one-measurement relation between the states of a physical system (Subsection 1.2); and the naive notion of indiscriminability gives rise to the following two hypotheses:

Hypothesis 1: A state can never be discriminated from itself.

Hypothesis 2: That a state $s$ is indiscriminable from a state $t$ means the same as that a state $t$ is indiscriminable from a state $s$.

These two hypotheses support Reflexivity and Symmetry in the definition of quantum Kripke frames.

In the remaining part of this section, for convenience, we fix an arbitrary physical system, classical or quantum, and consider a Kripke frame $\mathfrak{F}=(\Sigma, \rightarrow)$ that models its set of states and the indiscriminability relation between the states. As is discussed above, $\mathfrak{F}$ satisfies Reflexivity and Symmetry.

\subsection{Discussion on Representation (I): Closed Set}

In this subsection, we discuss about the antecedent of Representation.

We start from considering how to describe a measurement of an observable. Let $I$ be the non-empty set of all (mutually exclusive) possible values of an arbitrary observable $\mathcal{P}$ and, for each $i \in I, P_{i} \subseteq \Sigma$ be the set of states each of which certainly yields the value $i$ in a measurement of $\mathcal{P}$.

We propose the following hypothesis, which is based on what we find in experiments:

Hypothesis 3: A measurement of an observable must yield a value as an outcome. Once a measurement yields a value, any immediate repeat of this measurement will yield the same value.

According to this hypothesis, after a measurement of $\mathcal{P}$ yields a value $i \in I$, the state of the system will be an element of $P_{i}$.

As the model of an observable, $\left\{P_{i} \mid i \in I\right\}$ should satisfy two conditions.

First, $s \nrightarrow t$, for any distinct $i, j \in I, s \in P_{i}$ and $t \in P_{j}$. The reason is as follows: if we know that the state of the system is either $s$ or $t$, then the possible outcomes of a measurement of $\mathcal{P}$ will only be $i$ and $j$; moreover, the state is $s$ if and only if the outcome is $i$; and the state is $t$ if and only if the outcome is $j$. Hence $s$ and $t$ can be discriminated by one measurement of $\mathcal{P}$.

Note that this condition means that $\left\{P_{i} \mid i \in I\right\}$ is an opposite family. Also note that by Reflexivity $P_{i} \cap P_{j}=\emptyset$ holds for any distinct $i, j \in I$. With Hypothesis 3 this implies that no state can simultaneously yield two different values of an observable in one measurement, which is intuitive. 
Second, since $I$ is the set of all possible values of $\mathcal{P}$ and, for each $i \in I, P_{i}$ is the set of states each of which certainly yields the value $i$ in a measurement of $\mathcal{P}$, it makes sense to require that the opposite family $\left\{P_{i} \mid i \in I\right\}$ is maximal.

Remark 4.1. This mathematical condition of maximality is inherited from theories in physics, both classical and quantum, and I'm afraid that I do not have a very convincing argument for it. The following discussion may reveal a bit of its physical significance.

Consider the following statement:

(Max) An opposite family in $\mathfrak{F}$ describes an observable, if and only if it is maximal.

The 'if' part is a theoretical idealization about our experimental power which is arguably fine; the 'only if' part is in question.

For an opposite family $\left\{P_{i} \mid i \in I\right\}$ in $\mathfrak{F}$ that models an observable, $i^{*} \in I$ and $s \in \Sigma$, we consider the following two statements:

(i) $s \in \sim P_{i^{*}}$, i.e., for each $t \in P_{i^{*}}$, there is an observable such that one measurement of it can discriminate between $s$ and $t$;

(ii) a measurement of the observable modelled by $\left\{P_{i} \mid i \in I\right\}$ on the system in state $s$ never yields the value $i^{*}{ }^{18}$

I argue for 3 points.

First, I argue that, for any $\left\{P_{i} \mid i \in I\right\}, i^{*} \in I$ and $s \in \Sigma$, (ii) implies (i). Assume that $s \notin \sim P_{i^{*}}$, then $s \rightarrow t$ for some $t \in P_{i^{*}}$. Thus a measurement of the observable modelled by $\left\{P_{i} \mid i \in I\right\}$ can not discriminate between $s$ and $t$. Since by $t \in P_{i^{*}}$ a measurement in state $t$ certainly yields the value $i^{*}$, it must be possible for a measurement in state $s$ to yield the value $i^{*}$. In other words, a measurement in state $s$ may yield the value $i^{*}$.

Second, I argue that (Max) is sufficient for that, for any $\left\{P_{i} \mid i \in I\right\}, i^{*} \in I$ and $s \in \Sigma$, (i) implies (ii). Since $\left\{P_{i} \mid i \in I\right\}$ models an observable, by the 'only if' part of (Max) $\left\{P_{i} \mid i \in I\right\}$ is maximal, thus by Proposition 3.3 $P_{i^{*}}$ is closed. A measurement of the observable modelled by $\left\{P_{i} \mid i \in I\right\}$ can be considered as a measurement of another observable which has exactly two possible values, ' $i^{*}$ ' and 'not $i^{*}$, just by recording the results in a coarser way. By Proposition $3.3\left\{P_{i^{*}}, \sim P_{i^{*}}\right\}$ is a maximal opposite family in $\mathfrak{F}$. It follows from the 'if' part of (Max) that $\left\{P_{i^{*}}, \sim P_{i^{*}}\right\}$ models this coarse observable, and from the 'only if' part of (Max) that $\left\{P_{i^{*}}, \sim P_{i^{*}}\right\}$ is the unique opposite family that models this observable. Therefore, when $s \in \sim P_{i^{*}}$, it means that a measurement, which measures both of the observables, in state $s$ will certainly yield the value 'not $i^{*}$; in other words, the outcome $i^{*}$ never occurs in such a measurement.

Third, I argue that the 'only if' part of (Max) is necessary for that, for any $\left\{P_{i} \mid i \in I\right\}, i^{*} \in I$ and $s \in \Sigma$, (i) implies (ii). Suppose that an opposite family $\left\{P_{i} \mid i \in I\right\}$ in $\mathfrak{F}$ models an observable. I use Lemma 3.4. Let $i^{*} \in I$ be arbitrary. On the one hand, since $\left\{P_{i} \mid i \in I\right\}$ is an opposite family, by definition $P_{i^{*}} \subseteq \sim P_{i}$ holds for each $i \in I \backslash\left\{i^{*}\right\}$, thus $P_{i^{*}} \subseteq \bigcap\left\{\sim P_{i} \mid i \in I \backslash\left\{i^{*}\right\}\right\}$. On the other hand, for each $s \in \Sigma$, if $s \in \bigcap\left\{\sim P_{i} \mid i \in I \backslash\left\{i^{*}\right\}\right\}$, then by the implication from (i) to (ii) a measurement in state $s$ never yields any value in

\footnotetext{
${ }^{18}$ This means that, for each $t \in P_{i^{*}}$, a measurement of the observable modelled by $\left\{P_{i} \mid i \in\right.$ $I\}$ can discriminate between $s$ and $t$.
} 
$I \backslash\left\{i^{*}\right\}$. Since a measurement always yields a value in $I$, such a value will certainly be $i^{*}$, so $s \in P_{i^{*}}$. Therefore, $\bigcap\left\{\sim P_{i} \mid i \in I \backslash\left\{i^{*}\right\}\right\} \subseteq P_{i^{*}}$. As a result, $P_{i^{*}}=\bigcap\left\{\sim P_{i} \mid i \in I \backslash\left\{i^{*}\right\}\right\}$. By Lemma $3.4\left\{P_{i} \mid i \in I\right\}$ is maximal.

These three points together mean: (Max) is sufficient and necessary for that, for any observable with a set $I$ of possible values, $i^{*} \in I$ and $s \in \Sigma, s$ can be discriminated from each state which certainly yields the value $i^{*}$ in a measurement of the observable, if and only if a measurement of the observable on the state $s$ never yields the outcome $i^{*}$.

(Max), or the above condition equivalent to it, is an implicit theoretical assumption in physical theories.

We have seen that maximal opposite families in $\mathfrak{F}$ correspond to observables. When applied to quantum theory, this is quite restrictive, because such observables are modelled by compact self-adjoint operators. However, this is the core idea and can be generalized, as will be presented in Subsection 4.6.

Remark 4.2. In some cases, e.g. classical physics, it is reasonable to assume that any two distinct states can be discriminated. Then the binary relation $\rightarrow$ boils down to the graph of the identity map on $\Sigma$. In such cases, maximal opposite families in $\mathfrak{F}$ coincide with partitions of $\Sigma$. Therefore, the way how an observable is modelled here is a generalization of the way how a question is modelled in various logics of questions [7, 20].

Moreover, each $P_{i}$ in a maximal opposite family $\left\{P_{i} \mid i \in I\right\}$ of $\mathfrak{F}$ can be considered as a property of the system from the extensional view on properties, thus by Proposition 3.3 closed sets in $\mathfrak{F}$ correspond to properties of the system. This explains why such sets appear in Representation.

With the above discussion, we understand the antecedent of Representation: it is about a situation when a closed set $P$ contains exactly the states each of which will certainly yield the same particular value of an observable in a measurement, and the system is in such a state $s$ that a measurement of this observable is possible to yield this value.

\subsection{Discussion on Representation (II): Representative}

In this subsection, we discuss about the consequent of Representation.

By Hypothesis 3, if an observable modelled by $\left\{P_{i} \mid i \in I\right\}$ is measured on the system in a state $s \in \Sigma$ and the outcome is $i \in I$, then the state after this measurement is in $P_{i}$. Here we make an assumption of modelling that $\left\{P_{i} \mid i \in I\right\}, s$ and $i$ determine a unique state after measurement. In the following, I will argue that this state should be a representative of $s$ in $P_{i}$.

I need the notion of refinements of an observable:

Definition 4.3. Let $\mathcal{P}$ and $\mathcal{Q}$ be two observables with the sets of possible values $I$ and $J$, respectively. $\mathcal{Q}$ is a refinement of $\mathcal{P}$, if there is a surjection $f: J \rightarrow I$ such that, whenever a measurement of $\mathcal{Q}$ yields a value $j \in J$, an immediate measurement of $\mathcal{P}$ will certainly yield the value $f(j)$.

Correspondingly, we define refinements of a maximal opposite family:

Definition 4.4. A maximal opposite family $\left\{Q_{j} \mid j \in J\right\}$ in $\mathfrak{F}$ is a refinement of a maximal opposite family $\left\{P_{i} \mid i \in I\right\}$ in $\mathfrak{F}$, if there is a surjection $f: J \rightarrow I$ such that, for each $j \in J, Q_{j} \subseteq P_{f(j)}$. 
For two observables denoted by $\mathcal{P}$ and $\mathcal{Q}$, respectively, we denote by $\mathcal{P} ; \mathcal{Q}$ the experiment in which we first measure $\mathcal{P}$ and then measure $\mathcal{Q}$, and $\mathcal{Q} ; \mathcal{P}$ the experiment where the order is reversed. Consider the following hypothesis:

Hypothesis 4: For any two observables $\mathcal{P}$ and $\mathcal{Q}$ with the sets of possible values $I$ and $J$, respectively, such that $\mathcal{Q}$ is a refinement of $\mathcal{P}$, for any $i \in I$ and $j \in J$, for the system prepared in any state $s$, the result $(i, j)$ is possible in the experiment $\mathcal{P} ; \mathcal{Q}$, if and only if the result $(j, i)$ is possible in the experiment $\mathcal{Q} ; \mathcal{P}$.

This hypothesis is plausible, because $\mathcal{P}$ and $\mathcal{Q}$ can be measured by the same experimental apparatus but only different in the ways of recording the results.

We can argue that, to accord with Hypothesis 4, the following condition is sufficient:

(*) the state of the system after a measurement is a representative of the state before the measurement in the closed set corresponding to the outcome of the measurement.

Denote by $t$ the state after a measurement of the observable $\mathcal{P}$ modelled by $\left\{P_{i} \mid i \in I\right\}$ in the state $s$ with outcome $i^{*} \in I$. By Hypothesis $3 t \in P_{i^{*}}$.

Assume that $t$ is a representative of $s$ in $P_{i^{*}}$. Let $\mathcal{Q}$ be an observable modelled by $\left\{Q_{j} \mid j \in J\right\}$ which is a refinement of $\left\{P_{i} \mid i \in I\right\}$ witnessed by a surjection $f$ and $j^{*} \in J$ satisfying $f\left(j^{*}\right)=i^{*}$. If the result $\left(i^{*}, j^{*}\right)$ is possible in the experiment $\mathcal{P} ; \mathcal{Q}$, then by Remark $4.1 t \notin \sim Q_{j^{*}}$. Since $Q_{j^{*}} \subseteq P_{i^{*}}$ and $s \approx_{P_{i^{*}}} t$, $s \notin \sim Q_{j^{*}}$. Hence in the experiment $\mathcal{Q} ; \mathcal{P} j^{*}$ is a possible outcome of measuring $\mathcal{Q}$ and $i^{*}$ is certain to be the outcome of the measurement of $\mathcal{P}$. Therefore, the result $\left(j^{*}, i^{*}\right)$ is also possible in the experiment $\mathcal{Q} ; \mathcal{P}$. If the result $\left(j^{*}, i^{*}\right)$ is possible in the experiment $\mathcal{Q} ; \mathcal{P}$, then by Remark $4.1 s \notin \sim Q_{j^{*}}$. Since $Q_{j^{*}} \subseteq P_{i^{*}}$ and $s \approx_{P_{i^{*}}} t, s \notin \sim P_{i^{*}}$ and $t \notin \sim Q_{j^{*}}$. Hence in the experiment $\mathcal{P} ; \mathcal{Q} i^{*}$ is a possible outcome of measuring $\mathcal{P}$ and $j^{*}$ is a possible outcome of measuring $\mathcal{Q}$. Therefore, the result $\left(i^{*}, j^{*}\right)$ is also possible in the experiment $\mathcal{P} ; \mathcal{Q}$. In a word, a result is possible in one of the two experiments, if and only if it is possible in the other.

In fact, if $\mathfrak{F}$ satisfies Representation and Separation, to accord with Hypothesis 4 , the condition (*) is also necessary. This is similar to the justification of the Projection Postulate, and the idea here is similar to that of Bub in [11].

Our argument needs the following proposition, whose proof needs some definitions and results in the Appendix.

Proposition 4.5. Suppose that $\mathfrak{F}=(\Sigma, \rightarrow)$ satisfies Separation and Representation (in addition to Reflexivity and Symmetry). Let $s, t \in \Sigma,\left\{P_{i} \mid i \in I\right\}$ be a maximal opposite family in $\mathfrak{F}$ and $i^{*} \in I$. The following are equivalent:

(i) $s \approx_{P_{i *}} t$

(ii) for any refinement $\left\{Q_{j} \mid j \in J\right\}$ of $\left\{P_{i} \mid i \in I\right\}$ witnessed by a surjection $f: J \rightarrow I$ and $j \in J$ satisfying $f(j)=i^{*}, s \in \sim Q_{j}$ if and only if $t \in \sim Q_{j}$.

Proof. From (i) to (ii): Let $\left\{Q_{j} \mid j \in J\right\}$ be a maximal opposite family which is a refinement of $\left\{P_{i} \mid i \in I\right\}$ witnessed by a surjection $f: J \rightarrow I$, and $j \in J$ satisfying $f(j)=i^{*}$. Then by definition $Q_{j} \subseteq P_{i^{*}}$. It follows from $s \approx_{P_{i^{*}}} t$ that $s \in \sim Q_{j}$ if and only if $t \in \sim Q_{j}$. 
From (ii) to (i): Assume that (i) does not hold. Without loss of generality we assume that $I \cap\{0,1\}=\emptyset$. We consider two cases.

Case 1: There is a $w \in P_{i^{*}}$ such that $s \rightarrow w$ but $t \not \rightarrow w$. Consider the set $\left\{Q_{j} \mid j \in J\right\}$ with $J=\left(I \backslash\left\{i^{*}\right\}\right) \cup\{0,1\}$ defined as follows:

$$
Q_{j}= \begin{cases}\{w\}, & \text { if } j=0 \\ \sim\{w\} \cap P_{i^{*}}, & \text { if } j=1 \\ P_{j}, & \text { if } j \in I \backslash\left\{i^{*}\right\}\end{cases}
$$

By Lemma $2.8\{w\}$ is closed in $\mathfrak{F}$. By Lemma A.3 $\{w\}$ is closed in the subframe $\left(P_{i^{*}}, \rightarrow\right)$ of $\mathfrak{F}$. Then $\left(\{w\}, \sim\{w\} \cap P_{i^{*}}\right)=\left(\{w\}, \sim_{P_{i^{*}}}\{w\}\right)$ is a maximal opposite family in the subframe $\left(P_{i^{*}}, \rightarrow\right)$ of $\mathfrak{F}$ by Proposition 3.3 and Lemma A.3. Since $\left\{P_{i} \mid i \in I\right\}$ is a maximal opposite family in $\mathfrak{F}$, by Lemma A.7 $\left\{Q_{j} \mid j \in J\right\}$ is a maximal opposite family in $\mathfrak{F}$. It is obvious that $\{w\} \subseteq P_{i^{*}}$ and $\sim\{w\} \cap P_{i^{*}} \subseteq$ $P_{i^{*}}$, so $\left\{Q_{j} \mid j \in J\right\}$ is a refinement of $\left\{P_{i} \mid i \in I\right\}$ witnessed by the surjection $f=\left\{\left(0, i^{*}\right),\left(1, i^{*}\right)\right\} \cup\left\{(i, i) \mid i \in I \backslash\left\{i^{*}\right\}\right\}$. Then $f(0)=i^{*}, s \notin \sim Q_{0}$ but $t \in \sim Q_{0}$, so (ii) fails.

Case 2: There is a $w \in P_{i^{*}}$ such that $s \not \rightarrow w$ but $t \rightarrow w$. Construct a refinement $\left\{Q_{j} \mid j \in J\right\}$ of $\left\{P_{i} \mid i \in I\right\}$ and a witnessing surjection $f$ in the same way as the above case. Then $f(0)=i^{*}, s \in \sim Q_{0}$ but $t \notin \sim Q_{0}$, so (ii) fails.

With this proposition, we can argue for the necessity of the condition $(*)$, when $\mathfrak{F}$ satisfies Representation and Separation in addition.

Denote by $t$ the state after a measurement of the observable $\mathcal{P}$ modelled by $\left\{P_{i} \mid i \in I\right\}$ in a state $s$ with outcome $i^{*} \in I$. By Hypothesis $3 t \in P_{i^{*}}$.

Assume that $t$ is not the representative of $s$ in $P_{i^{*}}$. Then by the direction from (ii) to (i) in the above proposition we can find an observable $\mathcal{Q}$ modelled by $\left\{Q_{j} \mid j \in J\right\}$ which is a refinement of $\left\{P_{i} \mid i \in I\right\}$ witnessed by a surjection $f$ and a $j^{*} \in J$ satisfying $f\left(j^{*}\right)=i^{*}$ such that either (1) $s \in \sim Q_{j^{*}}$ and $t \notin \sim Q_{j^{*}}$ or (2) $s \notin \sim Q_{j^{*}}$ and $t \in \sim Q_{j^{*}}$. In Case (1), by Remark 4.1 the result $\left(i^{*}, j^{*}\right)$ is possible in the experiment $\mathcal{P}$; $\mathcal{Q}$ because $t \notin \sim Q_{j^{*}}$; but the result $\left(j^{*}, i^{*}\right)$ is not possible in the experiment $\mathcal{Q} ; \mathcal{P}$ because $s \in \sim Q_{j^{*}}$. In Case (2), by Remark 4.1 the result $\left(i^{*}, j^{*}\right)$ is not possible in the experiment $\mathcal{P} ; \mathcal{Q}$ because $t \in \sim Q_{j^{*}}$; but the result $\left(j^{*}, i^{*}\right)$ is possible in the experiment $\mathcal{Q} ; \mathcal{P}$ because $s \notin \sim Q_{j^{*}}$. In both cases, reversing the order affects the set of possible results of the experiment.

Through the above discussion, we understand the consequent of Representation: in the situation described by the antecedent, Representation guarantees that there is an element in the Kripke frame that can reasonably describe the state after the measurement.

The conclusion of this subsection is that Representation gives us a way that makes sense in physics to describe measurements of observables.

\subsection{Discussion on Separation}

Separation is a theoretical idealization. It means that the indiscriminability relation has enough power to separate any two distinct states. From the proof of Item 6 in Lemma 2.8 we see that, in a Kripke frame satisfying Reflexivity and Symmetry, Separation is equivalent to that every singleton is closed. Considering Proposition 3.3 and Remark 4.1, we propose the following hypothesis: 
Hypothesis 5: For each state $s$, there is an observable and a possible value of it such that $s$ is the unique state that certainly yields this value in a measurement of this observable.

Relaxing Separation may enable our relational framework to model situations that are more general. For example, Mr. Fangjun Hu (personal communication, April 2019) proves that, in a quantum system modelled by a finitedimensional Hilbert space over $\mathbb{C}$, the non-orthogonality relation between the mixed states satisfies Reflexivity, Symmetry, Representation and Superposition, but not Separation; where two mixed states, modelled by two density operators $\rho$ and $\rho^{\prime}$, respectively, are said to be non-orthogonal if $\operatorname{Tr}\left(\rho \rho^{\prime}\right) \neq 0$.

\subsection{Discussion on Superposition}

Superposition is an abstraction of the Superposition Principle in quantum theory, which we propose as a hypothesis here:

Hypothesis 6: For any two states, there is a state which can not be discriminated with any one of them in a measurement of any observable.

For example, the state in which an electron has a particular value of spin in the $x$-direction can not be discriminated with either the state of spin-up or the state of spin-down in the $z$-direction, no matter what observable we measure.

In this subsection, I will argue that Superposition is the only condition with characteristic from quantum physics; in other words, a Kripke frame satisfying Reflexivity, Symmetry, Separation and Representation can be used to model a classical system.

In classical physics it is a plausible physical idealization that any two distinct states can be discriminated by one measurement of some observable. Based on this intuition, I define classical frames as follows:

Definition 4.6. A classical frame is a Kripke frame $\mathfrak{F}=(\Sigma, \rightarrow)$ such that $\rightarrow$ is (the graph of) the identity map on $\Sigma$, i.e. $\rightarrow=I d_{\Sigma}=\{(s, t) \in \Sigma \times \Sigma \mid s=t\}$.

The following are some basic properties of classical frames.

Lemma 4.7. Let $\mathfrak{F}=(\Sigma, \rightarrow)$ be a classical frame.

1. $\sim P=\Sigma \backslash P$, for every $P \subseteq \Sigma$.

2. $P=\sim \sim P$, for every $P \subseteq \Sigma$.

3. $\mathfrak{F}$ satisfies Reflexivity, Symmetry, Separation and Representation.

The following proposition shows the difference between quantum Kripke frames and classical frames.

Proposition 4.8. Let $\mathfrak{F}=(\Sigma, \rightarrow)$ be a Kripke frame satisfying Reflexivity, Symmetry, Separation and Representation.

1. $\mathfrak{F}$ is a quantum Kripke frame, if and only if Superposition holds.

2. $\mathfrak{F}$ is a classical frame, if and only if Transitivity holds, i.e. $s \rightarrow t$ and $t \rightarrow u$ imply that $s \rightarrow u$, for any $s, t, u \in \Sigma$. 
3. Both Superposition and Transitivity hold, if and only if $\Sigma$ has exactly one element.

Proof. For Item 1: It follows directly from the definition.

For Item 2: The 'only if' part is obvious from the definition of classical frames. For the 'if' part, assume that Transitivity holds. Let $s, t \in \Sigma$ be arbitrary such that $s \rightarrow t$. Then, for each $w \in \Sigma, s \rightarrow w$ if and only if $t \rightarrow w$ by Symmetry and Transitivity. It follows from the contrapositive of Separation that $s=t$. Combining this with Reflexivity, I conclude that $\rightarrow=\{(s, t) \in$ $\Sigma \times \Sigma \mid s=t\}$. Therefore, $\mathfrak{F}$ is a classical frame.

For Item 3: The 'If' Part: Assume that $\Sigma$ has exactly one element, namely $s$. Then it can be verified that $\mathfrak{F}=(\{s\},\{(s, s)\})$, and thus both Superposition and Transitivity hold.

The 'Only If' Part: Assume that both Superposition and Transitivity hold. Let $s, t \in \Sigma$ be arbitrary. By Superposition there is a $w \in \Sigma$ such that $w \rightarrow s$ and $w \rightarrow t$. By Transitivity and $2 \mathfrak{F}$ is a classical frame. It follows that $s=w$ and $t=w$, and thus $s=t$. Since $\Sigma$ cannot be empty by definition, $\Sigma$ has exactly one element.

This proposition formally captures the idea that quantum theory is different from classical physics only because of the Superposition Principle.

\subsection{A Qualitative Quantum Theory}

The discussion above shows how the structure of a quantum Kripke frame can be used in modelling physical concepts. By replacing the structure in a Hilbert space with its counterpart in a quantum Kripke frame, we obtain the following qualitative version of quantum theory:

1. A quantum system is modelled by a quantum Kripke frame $\mathfrak{F}=(\Sigma, \rightarrow)$ in such a way that (pure) states of the systems correspond to elements of $\Sigma$.

2. An observable is modelled by a function $\nu: \sigma \rightarrow \mathcal{L}_{\mathfrak{F}}$, where $\sigma$ is an algebra of measurable subsets of $\mathbb{R}$, such that:

(a) $\nu(\emptyset)=\emptyset$ and $\nu(\mathbb{R})=\Sigma$;

(b) for each $\left\{E_{i} \mid i \in \mathbb{N}\right\} \subseteq \sigma$, if $E_{i} \cap E_{j}=\emptyset$ holds for any two distinct $i, j \in \mathbb{N}$, then $\left\{\nu\left(E_{i}\right) \mid i \in \mathbb{N}\right\}$ is a maximal opposite family in $\nu\left(\bigcup\left\{E_{i} \mid i \in \mathbb{N}\right\}\right)$;

(c) for any $E, E^{\prime} \in \sigma, \nu\left(E \cap E^{\prime}\right)=\nu(E) \cap \nu\left(E^{\prime}\right)$.

In a measurement of this observable, if the system is prepared in the state $s \in \Sigma$ and the result lies in $E \in \sigma$, then the state after the measurement is the representative of $s$ in $\nu(E)$ (Item 7 in Lemma 2.8).

3. Evolutions are described by isomorphisms on $\mathfrak{F}$ : if a process of evolution is described by an isomorphism $F$ and the state before the process is $s \in \Sigma$, then after the process the state is $F(s)$. 
Now a quantum test measures an observable which has exactly two distinct possible values. We denote them by 0 and 1 . In this special case, the function $\nu$ essentially boils down to a maximal opposite family $\left\{P_{0}, P_{1}\right\}$ in $\mathfrak{F}$ in such a way that, for each $E \in \sigma$,

$$
\nu(E)= \begin{cases}\Sigma & \text { if } 0 \in E \text { and } 1 \in E \\ P_{0} & \text { if } 0 \in E \text { and } 1 \notin E \\ P_{1} & \text { if } 0 \notin E \text { and } 1 \in E \\ \emptyset & \text { if } 0 \notin E \text { and } 1 \notin E\end{cases}
$$

If the quantum system is prepared in the state $s \in \Sigma$, the possible outcomes and the states after the test in different cases are shown in the following table:

\begin{tabular}{|c|c|c|}
\hline Cases & Possible Outcome(s) & State after the Test \\
\hline \hline$s \in P_{0}$ & 0 & the representative of $s$ in $P_{0}$, i.e. $s$ \\
\hline$s \in P_{1}$ & 1 & the representative of $s$ in $P_{1}$, i.e. $s$ \\
\hline \multirow{2}{*}{$s \notin P_{0} \cup P_{1}$} & 0 & the representative of $s$ in $P_{0}$ \\
\cline { 2 - 3 } & 1 & the representative of $s$ in $P_{1}$ \\
\hline
\end{tabular}

This gives a clear, qualitative description of a quantum test, and it could be easily generalized to the case of a measurement of any observable.

\section{Conclusion}

In this paper we argue that quantum Kripke frames are able to model a large part of the qualitative aspect of the structure formed by the states of quantum systems. There are two main evidences. One is Theorem 2.13 which characterizes the quantum Kripke frames induced by infinite-dimensional Hilbert spaces. The other are six hypotheses in physics that reveal the rationale behind the definition and a qualitative version of quantum theory which describes the character of measurements in quantum physics. Along the way, an implicit theoretical assumption in theories of physics is revealed (Remark 4.1); and the Superposition Principle is argued, in terms of a theorem (Proposition 4.8), to be sufficient for explaining the difference between quantum theory and classical physics.

There are many topics for further investigation. Here I briefly mention two. First, the qualitative framework of quantum Kripke frames can be naturally extended to a quantitative one by replacing the non-orthogonality relation with transition probabilities. This approach is intuitive and has been investigated in the literature $([28,38])$. Second, we can find for two quantum Kripke frames the counterpart of the tensor product of two Hilbert spaces to describe the entanglement of two quantum systems. This involves a lot of technical work, so I defer to a separate paper the details and the physical intuition of this description.

Acknowledgements. All technical results in Sections 2 and A.1 are from my $\mathrm{PhD}$ thesis [40] finished at the Institute for Logic, Language and Computation (ILLC), University of Amsterdam, in 2015. I am very grateful to my supervisors, Prof. Johan van Benthem, Dr. Alexandru Baltag and Prof. Sonja Smets, for their supervision and the detailed comments on my thesis which are invaluable in the writing of this paper. I am also very grateful to the members in my $\mathrm{PhD}$ 
committee, Dr. Nick Bezhanishvili, Prof. Robert Goldblatt, Prof. John Harding, Prof. Yde Venema, Prof. Ronald de Wolf and Prof. Mingsheng Ying, for their comments on my thesis which are very helpful in writing this paper. The results were also presented in many workshops and seminars, and I am very grateful to the audiences for the interesting discussion. I also thank very much the three anonymous reviewers for their helpful comments. Finally, my PhD research was funded by China Scholarship Council (CSC), and the writing of this paper is supported by the National Social Science Fund of China (No. 20CZX048).

\section{References}

[1] S. Abramsky and B. Coecke. A categorical semantics of quantum protocols. In Proceedings of the 19th IEEE conference on Logic in Computer Science (LiCS'04), pages 415-425. IEEE Press, 2004.

[2] D. Aerts. Quantum axiomatics. In K. Engesser, D. M. Gabbay, and D. Lehmann, editors, Handbook of Quantum Logic and Quantum Structures: Quantum Logic, pages 79-126. Elsevier B.V., 2009.

[3] D. Aerts and B. van Steirteghem. Quantum axiomatics and a theorem of M.P. Solèr. International Journal of Theoretical Physics, 39(3):497-502, 2000 .

[4] I. Amemiya and H. Araki. A remark on Piron's paper. Publications of the Research Institute for Mathematical Sciences, 2(3):423-427, 1966.

[5] A. Baltag and S. Smets. Complete axiomatizations for quantum actions. International Journal of Theoretical Physics, 44(12):2267-2282, 2005.

[6] E. Beltrametti and G. Cassinelli. The Logic of Quantum Mechanics. Encyclopedia of Mathematics and Its Applications. Addison-Wesley Publishing Company, 1981.

[7] J. van Benthem and S. Minică. Toward a dynamic logic of questions. Journal of Philosophical Logic, 41:633-669, 2012.

[8] S.K. Berberian. Introduction to Hilbert Space. Oxford University Press, 1961.

[9] A. Beutelspacher and U. Rosenbaum. Projective Geometry: From Foundations to Applications. Cambridge University Press, 1998.

[10] G. Birkhoff and J. von Neumann. The logic of quantum mechanics. The Annals of Mathematics, 37:823-843, 1936.

[11] J. Bub. Quantum logic, conditional probability, and interference. Philosophy of Science, 49(3):402-421, 1982.

[12] M.L. Dalla Chiara, R. Giuntini, and R. Greechie. Reasoning in Quantum Theory: Sharp and Unsharp Quantum Logics, volume 22 of Trends in logic. Kluwer Acadamic Press, Dordrecht, 2004. 
[13] B. Coecke and A. Kissinger. Picturing Quantum Processes: A First Course in Quantum Theory and Diagrammatic Reasoning. Cambridge University Press, 2017.

[14] G.M. D'Ariano, G. Chiribella, and P. Perinotti. Quantum Theory from First Principles: An Informational Approach. Cambridge University Press, 2017.

[15] H. Dishkant. Semantics of the minimal logic of quantum mechanics. Studia Logica, 30(1):23-30, 1972.

[16] C.-A. Faure and A. Frölicher. Modern Projective Geometry. Springer Netherlands, 2000.

[17] D.J. Foulis and C.H. Randall. Lexicographic orthogonality. Journal of Combinatorial Theory, Series A, 11(2):157-162, 1971.

[18] R.I. Goldblatt. Semantic analysis of orthologic. Journal of Philosophical Logic, 3:19-35, 1974.

[19] R.I. Goldblatt. The Stone space of an ortholattice. Bulletin of the London Mathematical Society, 7(1):45-48, 1975.

[20] J. Groenendijk and M. Stokhof. Questions. In J. van Benthem and A. ter Meulen, editors, Handbook of Logic and Language, pages 1059-1131. Elsevier B.V., 2nd edition, 2011.

[21] B.C. Hall. Quantum Theory for Mathematicians. Springer New York, 2013.

[22] P.R. Halmos. Introduction to Hilbert Space and the Theory of Spectral Multiplicity. Chelsea Publishing Company, 1951.

[23] J. Hedlíková and S. Pulmannová. Orthogonality spaces and atomistic orthocomplemented lattices. Czechoslovak Mathematical Journal, 41:8-23, 1991.

[24] S.S. Holland. Orthomodularity in infinite dimensions: A theorem of M. Solèr. Bull. Amer. Math. Soc., 32:205-234, 1995.

[25] R.V. Kadison and J.R. Ringrose. Fundamentals of the Theory of Operator Algebras, Volumn I: Elementary Theory. American Mathematical Society, 1997.

[26] G. Kalmbach. Orthomodular Lattices. Acadamic Press, 1983.

[27] R. Mayet. Some characterizations of the underlying division ring of a Hilbert lattice by automorphisms. International Journal of Theoretical Physics, 37:109-114, 1998.

[28] B. Mielnik. Geometry of quantum states. Communications in Mathematical Physics, 9(1):55-80, 1968.

[29] P. Mittelstaedt. Quantum Logic. D. Reidel Publishing Company, 1978.

[30] P. Mittelstaedt. Rational Reconstructions of Modern Physics. Springer Netherlands, 2nd enlarged edition, 2013. 
[31] D.J. Moore. Categories of representations of physical systems. Helv Phys Acta, 68:658-678, 1995.

[32] C. Piron. Foundations of Quantum Physics. W.A. Benjamin Inc., Reading, 1976.

[33] R. Shankar. Principles of Quantum Mechanics. Plenum Press, second edition, 2008.

[34] M.P. Solèr. Characterization of Hilbert spaces with orthomodularity spaces. Communications in Algebra, 23:219-243, 1995.

[35] I. Stubbe and B. van Steirteghem. Propositional systems, Hilbert lattices and generalized Hilbert spaces. In K. Engesser, D.M. Gabbay, and D. Lehmann, editors, Handbook of Quantum Logic and Quantum Structures: Quantum Structures, pages 477-523. Elsevier B.V., 2007.

[36] J. von Neumann. Mathematische Grundlagen der Quantenmechanik. Julius Springer, 1932.

[37] E.P. Wigner. Group Theory and Its Application to the Quantum Mechanics of Atomic Spectra. Academic Press Inc., expanded and improved edition, 1959. translated from the German by J.J. Griffin.

[38] P. Zabey. Reconstruction theorems in quantum mechanics. Foundations of Physics, 5(2):323-342, 1975.

[39] C. Zhang, Y. Feng, and M. Ying. Unambiguous discrimination of mixed quantum states. Physics Letters A, 353(4):300-306, 2006.

[40] S. Zhong. Orthogonality and Quantum Geometry: Towards a Relational Reconstruction of Quantum Theory. PhD thesis, University of Amsterdam, 2015. http://www.illc.uva.nl/Research/Publications/Dissertations/DS2015-03.text.pdf.

[41] S. Zhong. A formal state-property duality in quantum logic. Studies in Logic, 10(2):112-133, 2017.

[42] S. Zhong. Correspondence between Kripke frames and projective geometries. Studia Logica, 106:167-189, 2018.

\section{A Appendix}

In this appendix, I will develop the technicalities used in the proof of Proposition 4.5 in Subsection 4.3.

We fix a Kripke frame $\mathfrak{F}=(\Sigma, \rightarrow)$ satisfying Reflexivity and Symmetry throughout this appendix for convenience.

Note that Items 1 to 5 in Lemma 2.8 only need Reflexivity and Symmetry in their proofs, so they hold in $\mathfrak{F}$. 


\section{A.1 Subframe}

In this subsection, we introduce and discuss the notion of a subframe.

Definition A.1. A subframe of $\mathfrak{F}$ is an ordered pair $\left(P, \rightarrow_{P}\right)$ such that

1. $P \subseteq \Sigma$ is non-empty and closed in $\mathfrak{F}$;

2. $\rightarrow_{P}$ is the restriction of $\rightarrow$ to $P$, i.e. $\rightarrow_{P}=\rightarrow \cap(P \times P)$.

Remark A.2. Let $\left(P, \rightarrow_{P}\right)$ be a subframe of $\mathfrak{F}$.

1. For any $s, t \in P, s \rightarrow t$ if and only if $s \rightarrow_{P} t$

2. For any $A \subseteq P$ and $s, t \in P, s$ and $t$ are indistinguishable with respect to $A$ in $\left(P, \rightarrow_{P}\right)$ if and only if they are indistinguishable with respect to $A$ in $\mathfrak{F}$.

3. $\left(P, \rightarrow_{P}\right)$ is a Kripke frame satisfying Reflexivity and Symmetry, so the results in this appendix apply to it as well.

Given this remark, in the following, I denote the restriction of $\rightarrow$ in $\mathfrak{F}$ to $P$ by $\rightarrow$ instead of $\rightarrow_{P}$. I also use the same symbol $\approx_{A}$ indexed by $A \subseteq P$ to denote the indistinguishability relation with respect to $A$ in $(P, \rightarrow)$ and that in $\mathfrak{F}$. On the contrary, I denote the orthocomplement of $A \subseteq P$ in $(P, \rightarrow)$ by $\sim_{P} A$, because the orthocomplements in the subframe are different from those in $\mathfrak{F}$.

I start from some simple facts about the orthocomplements and the closed subsets in a subframe.

Lemma A.3. Let $(P, \rightarrow)$ be a subframe of $\mathfrak{F}$.

1. $\sim_{P} A=\sim A \cap P$, for every $A \subseteq P$

2. For each $A \subseteq P$, if $\sim_{P} \sim_{P} A=A$, then $\sim \sim A=A$.

3. If $\mathfrak{F}$ satisfies Representation, then, for each $A \subseteq P, \sim \sim A=A$ implies that $\sim_{P} \sim_{P} A=A$

Proof. For Item 1: Easy verification.

For Item 2: Assume that $\sim_{P} \sim_{P} A=A$. By Lemma $2.8 A \subset \sim \sim A$. It remains to show that $\sim A \subseteq A$. Assume that $s \notin A$. By the assumption $s \notin \sim_{P} \sim_{P} A$. Then there is a $t \in \sim_{P} A$ such that $s \rightarrow t$. By $1 t \in \sim A \cap P$. Hence $t \in \sim A$ is such that $s \rightarrow t$, and thus $s \notin \sim \sim A$.

For Item 3: Assume that $\mathfrak{F}$ satisfies Representation and $\sim \sim A=A$. By 1 $\sim_{P} \sim_{P} A=\sim(\sim A \cap P) \cap P$, so it amounts to show that $\sim(\sim A \cap P) \cap P=A$.

On the one hand, since $A \subseteq P$ and $A=\sim \sim A \subseteq \sim(\sim A \cap P)$ by Lemma 2.8, $A \subseteq \sim(\sim A \cap P) \cap P$

On the other hand, let $s \in \sim(\sim A \cap P) \cap P$ be arbitrary. Then $s \in P$ and $s \in \sim(\sim A \cap P)$. To show that $s \in A$, by the assumption it suffices to show that $s \in \sim \sim A$. Hence we let $t \in \sim A$ be arbitrary and try to show that $s \not \rightarrow t$. Consider two cases.

- Case 1: $t \in \sim P$.

Since $s \in P$, it follows that $s \not \rightarrow t$. 
- Case 2: $t \notin \sim P$.

By Representation there is a $t_{\|} \in P$ such that $t \approx_{P} t_{\|}$. Since $A \subseteq P$ and $t \in \sim A, t_{\|} \in \sim A$. Hence $t_{\|} \in P \cap \sim A$. Since $s \in \sim(\sim A \cap P), t_{\|} \not \rightarrow s$. Since $s \in P$, by $t \approx_{P} t_{\|}$we have $t \nrightarrow \rightarrow s$, so $s \not \rightarrow t$.

Proposition A.4. Let $(P, \rightarrow)$ be a subframe of $\mathfrak{F}$. If $\mathfrak{F}$ satisfies both Separation and Representation, then so is $(P, \rightarrow)$.

Proof. For Representation, let $A \subseteq P$ and $s \in P$ be such that $A=\sim_{P} \sim_{P} A$ and $s \notin \sim_{P} A$. By Lemma A.3 $A=\sim \sim A$. Moreover, since $s \notin \sim_{P} A$ and $s \in P$, by Lemma A.3 $s \notin \sim A$. By Representation there is an $s^{\prime} \in A$ such that $s \approx_{A} s^{\prime}$.

For Separation, assume that $s, t \in P$ are such that $s \neq t$. Since $\mathfrak{F}$ satisfies Separation, there is a $w \in \Sigma$ such that $w \rightarrow s$ and $w \not t t$. Since $w \rightarrow s, w \notin \sim P$. By Representation there is a $w^{\prime} \in P$ such that $w \approx_{P} w^{\prime}$. Hence $w^{\prime} \in P$ is such that $w^{\prime} \rightarrow s$ and $w^{\prime} \not t t$.

\section{A.2 Maximal Opposite Family}

Here we prove some useful results about maximal opposite families.

Corollary A.5. Let $\left\{P_{i} \mid i \in I\right\}$ be a maximal opposite family in $\mathfrak{F}$. $\emptyset=$ $\bigcap\left\{\sim P_{i} \mid i \in I\right\}$.

Proof. If $I$ is a singleton, the result holds by Lemma 2.8 and Remark 3.2. In the following we focus on the case when $I$ has at least two elements.

Take an $i^{*} \in I$. By Lemma $3.4 P_{i^{*}}=\bigcap\left\{\sim P_{i} \mid i \in I \backslash\left\{i^{*}\right\}\right\}$. Hence $\bigcap\left\{\sim P_{i} \mid i \in I\right\}=\sim P_{i^{*}} \cap \bigcap\left\{\sim P_{i} \mid i \in I \backslash\left\{i^{*}\right\}\right\}=\sim P_{i^{*}} \cap P_{i^{*}}=\emptyset$.

Lemma A.6. If $\mathfrak{F}$ satisfies Separation and Representation, then, for any closed $P \varsubsetneqq \Sigma$ and $s \notin \sim P$, there are $s_{\|} \in P$ and $s_{\perp} \in \sim P$ such that $s_{\|} \in \sim \sim\left\{s, s_{\perp}\right\}$.

Proof. Assume that $P \varsubsetneqq \Sigma$ is closed and $s \notin \sim P$. Note that $\sim P \neq \emptyset$ for $P \neq \Sigma$. If $s \in P$, then by just letting $s_{\|}=s$ and picking an arbitrary $s_{\perp} \in \sim P$ we will have $s_{\|} \in \sim \sim\left\{s, s_{\perp}\right\}$. In the following, we focus on the case when $s \notin P$.

Since $P$ is closed, $s \notin \sim \sim P$. By Representation there is an $s_{\perp} \in \sim P$ such that $s \approx \sim P s_{\perp}$. Since $s \notin \sim P, s \neq s_{\perp}$, so $s \notin \sim \sim\left\{s_{\perp}\right\}$ by Separation and Lemma 2.8. By Representation there is an $s_{\|} \in \sim\left\{s_{\perp}\right\}$ such that $s \approx_{\sim\left\{s_{\perp}\right\}} s_{\|}$. It follows that $s_{\|} \in \sim \sim\left\{s, s_{\perp}\right\}$.

It remains to show that $s_{\|} \in P$. Suppose (towards a contradiction) that $s_{\|} \notin P$. Then $s_{\|} \notin \sim \sim P$. By Representation there is an $s_{\|}^{\prime} \in \sim P$ such that $s_{\|} \approx_{\sim P} s_{\|}^{\prime}$. One the one hand, since $s_{\|}^{\prime} \rightarrow s_{\|}^{\prime}, s_{\|} \rightarrow s_{\|}^{\prime}$. On the other hand, since $s_{\|} \nrightarrow s_{\perp}$ and $s_{\perp} \in \sim P, s_{\|}^{\prime} \nrightarrow s_{\perp}$. Since $s \approx_{\sim P} s_{\perp}$ and $s_{\|}^{\prime} \in \sim P, s \not \rightarrow s_{\|}^{\prime}$. Therefore, $s_{\|}^{\prime} \in \sim\left\{s, s_{\perp}\right\}$. Since $s_{\|} \in \sim \sim\left\{s, s_{\perp}\right\}, s_{\|} \not \rightarrow s_{\|}^{\prime}$. As a result, we have got a contradiction.

Finally, we prove a lemma for building maximal opposite families used in the proof of Proposition 4.5. 
Lemma A.7. Suppose that $\mathfrak{F}$ satisfies Separation and Representation. Let I and $J$ be two disjoint, non-empty sets, $\left\{P_{i} \mid i \in I\right\}$ a maximal opposite family in $\mathfrak{F}, i^{*} \in I,\left\{Q_{j} \mid j \in J\right\}$ a maximal opposite family in the subframe $\left(P_{i^{*}}, \rightarrow\right)$ of $\mathfrak{F}$ and $K=\left(I \backslash\left\{i^{*}\right\}\right) \cup J$. The following element in $\wp(\wp(\Sigma) \backslash\{\emptyset\})$ is a maximal opposite family in $\mathfrak{F}$ : for each $k \in K$,

$$
O_{k}= \begin{cases}P_{k}, & \text { if } k \in I \backslash\left\{i^{*}\right\} \\ Q_{k}, & \text { if } k \in J\end{cases}
$$

Proof. The case when $I=\left\{i^{*}\right\}$ follows from Remark 3.2. It remains to consider the case when $I$ is not a singleton.

In this case, it can be verified that $\left\{O_{k} \mid k \in K\right\}$ is an opposite family.

Before showing maximality, I claim that $\sim P_{i^{*}}=\bigcap\left\{\sim Q_{j} \mid j \in J\right\}$ : For one direction, for each $j \in J, Q_{j} \subseteq P_{i^{*}}$, so $\sim P_{i^{*}} \subseteq \sim Q_{j}$. Hence $\sim P_{i^{*}} \subseteq$ $\bigcap\left\{\sim Q_{j} \mid j \in J\right\}$. For the other direction, suppose (towards a contradiction) that $\bigcap\left\{\sim Q_{j} \mid j \in J\right\} \nsubseteq \sim P_{i^{*}}$. On the one hand, there is an $s \in \bigcap\left\{\sim Q_{j} \mid j \in J\right\}$ but $s \notin \sim P_{i^{*}}$. By Lemma A.6 there are $s_{\|} \in P_{i^{*}}$ and $s_{\perp} \in \sim P_{i^{*}}$ such that $s_{\|} \in \sim \sim\left\{s, s_{\perp}\right\}$. Since $s \in \bigcap\left\{\sim Q_{j} \mid j \in J\right\}$ and $s_{\perp} \in \sim P_{i^{*}} \subseteq \bigcap\left\{\sim Q_{j} \mid j \in J\right\}$, $s_{\|} \in \bigcap\left\{\sim Q_{j} \mid j \in J\right\}$. Thus $s_{\|} \in P_{i^{*}} \cap \bigcap\left\{\sim Q_{j} \mid j \in J\right\}$. On the other hand, since $\left\{Q_{j} \mid j \in J\right\}$ is a maximal opposite family in the subframe $\left(P_{i^{*}}, \rightarrow\right)$ of $\mathfrak{F}$, by Lemma A.3 and Corollary A.5 $\emptyset=\bigcap\left\{\sim_{P_{i^{*}}} Q_{j} \mid j \in J\right\}=\bigcap\left\{P_{i^{*}} \cap \sim Q_{j} \mid j \in\right.$ $J\}=P_{i^{*}} \cap \bigcap\left\{\sim Q_{j} \mid j \in J\right\}$, which contradicts that $s_{\|} \in P_{i^{*}} \cap \bigcap\left\{\sim Q_{j} \mid j \in J\right\}$. As a result, $\sim P_{i^{*}}=\bigcap\left\{\sim Q_{j} \mid j \in J\right\}$.

Now we prove maximality. By Lemma 3.4 it suffices to show that, for each $k^{*} \in K, O_{k^{*}}=\bigcap\left\{\sim O_{k} \mid k \in K \backslash\left\{k^{*}\right\}\right\}$. We consider 2 cases.

- Case 1: $k^{*} \in I \backslash\left\{i^{*}\right\}$.

$$
\begin{array}{rll}
O_{k^{*}}=P_{k^{*}} & =\bigcap\left\{\sim P_{i} \mid i \in I \backslash\left\{k^{*}\right\}\right\} & \text { (Lemma 3.4) } \\
& =\sim P_{i^{*}} \cap \bigcap\left\{\sim P_{i} \mid i \in I \backslash\left\{k^{*}, i^{*}\right\}\right\} & \\
& =\bigcap\left\{\sim Q_{j} \mid j \in J\right\} \cap \bigcap\left\{\sim P_{i} \mid i \in I \backslash\left\{k^{*}, i^{*}\right\}\right\} & \text { (the claim) } \\
& =\bigcap\left\{\sim O_{k} \mid k \in K \backslash\left\{k^{*}\right\}\right\} &
\end{array}
$$

- Case 2: $k^{*} \in J$.

$$
\begin{aligned}
O_{k^{*}}=Q_{k^{*}} & =\bigcap\left\{\sim_{P_{i^{*}}} Q_{j} \mid j \in J \backslash\left\{k^{*}\right\}\right\} \\
& =\bigcap\left\{\sim Q_{j} \cap P_{i^{*}} \mid j \in J \backslash\left\{k^{*}\right\}\right\} \\
& =P_{i^{*}} \cap \bigcap\left\{\sim Q_{j} \mid j \in J \backslash\left\{k^{*}\right\}\right\} \\
& =\bigcap\left\{\sim P_{i} \mid i \in I \backslash\left\{i^{*}\right\}\right\} \cap \bigcap\left\{\sim Q_{j} \mid j \in J \backslash\left\{k^{*}\right\}\right\} \\
& =\bigcap\left\{\sim O_{k} \mid k \in K \backslash\left\{k^{*}\right\}\right\}
\end{aligned}
$$

